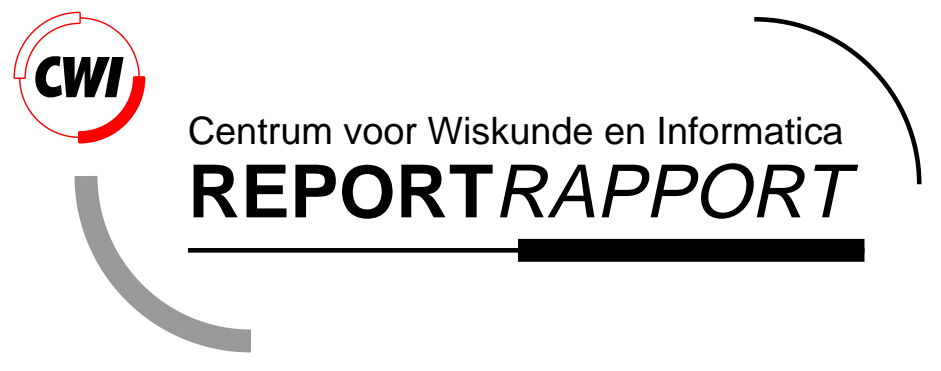

Spatial Discretization of the Shallow Water Equations in Spherical Geometry using Osher's Scheme

D. Lanser, J.G. Blom, J.G. Verwer

Modelling, Analysis and Simulation (MAS)

MAS-R9918 July 31, 1999 
Report MAS-R9918

ISSN 1386-3703

CWI

P.O. Box 94079

1090 GB Amsterdam

The Netherlands

$\mathrm{CWI}$ is the National Research Institute for Mathematics and Computer Science. CWI is part of the Stichting Mathematisch Centrum (SMC), the Dutch foundation for promotion of mathematics and computer science and their applications.

SMC is sponsored by the Netherlands Organization for Scientific Research (NWO). CWI is a member of ERCIM, the European Research Consortium for Informatics and Mathematics.

Copyright (C) Stichting Mathematisch Centrum P.O. Box 94079, 1090 GB Amsterdam (NL) Kruislaan 413, 1098 SJ Amsterdam (NL) Telephone +3120 5929333 Telefax +31205924199 


\title{
Spatial Discretization of the Shallow Water Equations in Spherical Geometry using Osher's Scheme
}

\author{
D. Lanser, J.G. Blom and J.G. Verwer \\ $C W I$ \\ P.O. Box 94079, 1090 GB Amsterdam, The Netherlands
}

debby.lanser@cwi.nl, joke.blom@cwi.nl, jan.verwer@cwi.nl

\begin{abstract}
The shallow water equations in spherical geometry provide a first prototype for developing and testing numerical algorithms for atmospheric circulation models. Since the seventies these models are often solved with spectral methods. Increasing demands on grid resolution combined with massive parallelism and local grid refinement seem to offer significantly better perspectives for gridpoint methods. In this paper we study the use of Osher's finite-volume scheme for the spatial discretization of the shallow water equations on the rotating sphere. This high-order finite volume scheme of upwind type is well suited to solve a hyperbolic system of equations. Special attention is paid to the pole problem. To that end Osher's scheme is applied on the common (reduced) latitude-longitude grid and on a stereographic grid. The latter is most appropriate in the polar region as in stereographic coordinates the pole singularity does not exist. The latitude-longitude grid is preferred on lower latitudes. Therefore, across the sphere we apply Osher's scheme on a combined grid connecting the two grids at high latitude. We will show that this provides an attractive spatial discretization for explicit integration methods, as it can greatly reduce the time step limitation incurred by the pole singularity when using a latitude-longitude grid only. When time step limitation plays no significant role, the standard (reduced) latitude-longitude grid is advocated provided that the grid is kept sufficiently fine in the polar region to resolve flow over the poles.
\end{abstract}

1991 Mathematics Subject Classification: 65M06, 86-08, 86A10.

1991 ACM Computing Classification System: G.1.8, J.2.

Keywords and Phrases: Numerical solution of PDEs, Atmospheric flow, SWEs in spherical geometry, Osher's scheme, stereographic coordinates.

Note: Work carried out under project MAS1.4 - Exploratory research: Discretization of initial value problems.

\section{INTRODUCTION}

For long people have tried to forecast the weather, first by observation of current and historical meteorological data, later by numerical simulation with circulation models based on the atmospheric primitive equations $[4,9,12,13]$. Nowadays circulation models are wide-spread. Besides being used in weather forecasting, they are applied as climate simulation models and provide meteorological input data needed in air pollution descriptions.

During the sixties the field of frequently used approximation methods in circulation models mainly consisted of gridpoint methods. In 1970 this accent shifted when Orszag and Eliasen et al. $[6,14]$ introduced the spectral transform method in global atmospheric modelling. Because spectral methods proved to be very accurate and cost efficient, they started to dominate the field of approximation methods used in global atmospheric modelling. Recently though the discussion on numerical methods applicable in circulation models has been renewed. Spectral methods are no longer considered ideal. Progression in atmospheric modelling, on the meteorological as well as on the computational side, demands higher grid resolutions than in the past. At high resolutions improved gridpoint methods can compete with the spectral ones on efficiency grounds, as is also stated in [5]. Furthermore, the global property of the spectral methods has shown some drawbacks. Although this property contributes highly to the accuracy of the found solution, it leads to 
inconveniences when one tries to parallelize spectral codes on parallel machines with distributed memory.

In this paper we develop a new numerical gridpoint method. Following [3, 26] and others we consider the 2D shallow water equations (SWEs) on the rotating sphere, which serve as a first prototype for a circulation model. The SWEs describe the behavior of a shallow homogeneous incompressible and inviscid fluid layer. Although in comparison to the full set of atmospheric primitive equations, these equations are obviously incomplete, they present some of the major difficulties associated with the horizontal dynamical aspects of circulation models on the earth. To avoid the well-known pole problem [19], which arises when a gridpoint method is applied on a full latitude-longitude grid, we study a combined grid composed of a (reduced) lat-lon grid away from the poles and a stereographic grid at the two polar caps. The use of the stereographic grid has been proposed before by Phillips and Browning $[2,17]$. Furthermore, to respect the underlying physical conservation laws and the characteristic directions associated with the hyperbolic character of our equations, we apply a finite volume method of upwind type. Within this group of methods Osher's approximate Riemann solver makes a good choice. First, it is robust and higher order accurate when combined with the right state interpolation. Second, from a future perspective, it has a logical extension to more realistic primitive equations and a nice boundary treatment, which puts Osher in favor before for instance a Roe solver. Finally, our upwind scheme is a scheme of flux difference splitting type (FDS). Schemes of flux vector splitting type (FVS) do not provide an alternative in this case, since the necessary condition for these schemes, i.e., that the Jacobian of the flux vector is homogeneous of degree one, is not fulfilled. For a detailed description of FDS and FVS methods we refer to [11].

In Section 2 we focus on the formulation of the SWEs in the two different coordinate systems. In Section 3.1 we attend to the construction of our combined grid. The spatial discretization of the equations, i.e. a description of our finite volume method, is given in Section 3.2. Special attention is paid to the connection problem, which occurs at the grid interface, when coupling the spherical grid part with the stereocaps. Numerical results from calculations on combined grids and on fully lat-lon grids are given in Section 4. Calculations are done on test case two of the testset in [26], which is a standard to test new numerical methods for solving the SWEs in spherical geometry. Test case two provides us with a good non-linear test to evaluate the scheme's ability to handle the poles. The main conclusions of our investigations are formulated in Section 5. These conclusions will confirm our objective, that is to show that on high resolution grids, a finite-volume scheme like Osher's applied on a lat-lon or on a combined grid provides an accurate and efficient method to solve the SWEs in spherical geometry.

\section{The Shallow Water equations}

Since they cover important aspects of the horizontal dynamical behavior of the atmosphere, the SWEs on the sphere suffice as a first prototype of a circulation model. Through the laws of conservation of mass and momentum, the SWEs on the sphere can be derived to describe the behavior (velocities and fluid depth) of a shallow homogeneous incompressible and inviscid fluid layer on the earth. In other words, we assume that the atmosphere can be regarded as a thin layer of air in which the density is uniform and constant, and viscous effects can be ignored. By using the SWEs, it is further assumed that the velocity component normal to the earth surface, the vertical component, is zero and that the vertical component of the Coriolis acceleration can be neglected in comparison with gravity. The centrifugal force is also neglected. The SWEs then follow from the Navier Stokes equations on the rotating sphere by integration over the fluid depth (depth-averaging), for details see [9]. A derivation of more realistic atmospheric primitive equations can be found in $[9,12]$.

\subsection{The shallow water equations in spherical coordinates}

Let $(\lambda, \phi, t)$ denote the independent variables longitude $(\lambda \in[0,2 \pi))$, latitude $\left(\phi \in\left[-\frac{\pi}{2},+\frac{\pi}{2}\right]\right)$ and time $(t \geq 0)$. Let $u$ be the velocity in the longitudinal direction, $v$ the velocity in the latitudinal direction and $H$ the depth of the fluid layer. Let $h$ be the height of the free surface above the sphere at sea level, $h=H+h_{s}$, where $h_{s}$ accounts for the orography of the earth associated 
with the height of mountains. Further, let $\underline{u}$ denote the horizontal velocity field $(u, v)$ defined by $u=a \cos \phi \frac{\mathrm{d} \lambda}{\mathrm{d} t}$ and $v=a \frac{\mathrm{d} \phi}{\mathrm{d} t}$. Let $f$ denote the Coriolis parameter $2 \Omega \sin \phi$ with $\Omega$ the angular velocity of the earth, $a$ the radius of the earth and $g$ the gravitational constant. The SWEs on the sphere in flux-form can then be formulated as

$$
\begin{aligned}
\frac{\partial H}{\partial t}+\nabla \cdot(H \underline{u}) & =0, \\
\frac{\partial H u}{\partial t}+\nabla \cdot(H u \underline{u}) & =\left(f+\frac{u}{a} \tan \phi\right) H v-\frac{g H}{a \cos \phi} \frac{\partial h}{\partial \lambda}, \\
\frac{\partial H v}{\partial t}+\nabla \cdot(H v \underline{u}) & =-\left(f+\frac{u}{a} \tan \phi\right) H u-\frac{g H}{a} \frac{\partial h}{\partial \phi},
\end{aligned}
$$

where the divergence operator is defined by

$$
\nabla \cdot \underline{u} \equiv \frac{1}{a \cos \phi}\left[\frac{\partial u}{\partial \lambda}+\frac{\partial v \cos \phi}{\partial \phi}\right] .
$$

The right-hand side in the momentum equations (2.2) and (2.3) represents respectively the Coriolis force, the hydrostatical pressure gradient force and an additional term due to the relative motion in the rotating coordinate system in longitudinal and latitudinal direction, see [12].

\subsection{The shallow water equations in stereographic coordinates}

The spherical formulation of the SWEs (2.1)-(2.3) has the disadvantage that is singular at the poles. To circumvent this problem, we formulate the SWEs in the stereographic coordinate system. In contrast to the formulation in spherical coordinates $(\lambda, \phi)$, this formulation reveals no singularities in the poles. We note that the stereographic projection is conformal, so the general form of the equations is preserved.

The stereographic projection in terms of the latitude-longitude coordinates is defined by

$$
\begin{aligned}
& x_{\mathrm{st}}=a m \cos \phi \cos \lambda, \\
& y_{\mathrm{st}}=a m \cos \phi \sin \lambda,
\end{aligned}
$$

where $m$ is the map factor

$$
m=\frac{2}{1+\alpha \sin \phi},
$$

with $\alpha$ distinguishing between the northern $(\alpha=1)$ and the southern hemisphere projection $(\alpha=-1)$. The poles are directly projected onto the origin of the stereographic planes. The northern hemisphere is projected from the south pole onto the northern stereographic plane, which is the plane locally tangent to the sphere at the north pole, see Figure 1. Likewise, the southern hemisphere is projected from the north pole onto the southern stereographic plane, which is locally tangent to the sphere at the south pole. A description of the construction of the stereographic projection is given in Appendix A. Note that the positive stereographic $x_{\mathrm{st}}$-axis for both the northern and southern hemisphere corresponds with the intersection of the half-plane $S_{\lambda=0}$ and the corresponding stereographic plane. Likewise, the positive stereographic $y_{\mathrm{st}}$-axis corresponds, for both hemispheres, with the intersection of the half-plane $S_{\lambda=\pi / 2}$ and the corresponding stereographic plane. Before we give the SWEs in the stereographic formulation, as found for instance in $[2,17,25]$, we need to define the velocity field in the new stereographic coordinate system. Let $\underline{U}=(U, V)$ be the velocity field in stereographic coordinates with $U$ the velocity in the $x_{\mathrm{st}}$-direction and $V$ the velocity in $y_{\text {st }}$-direction. We have

$$
\underline{U}=\left(\begin{array}{c}
U \\
V
\end{array}\right)=\left(\begin{array}{c}
m^{-1} \frac{\mathrm{d} x_{\mathrm{st}}}{\mathrm{d} t} \\
m^{-1} \frac{\mathrm{d} y_{\mathrm{st}}}{\mathrm{d} t}
\end{array}\right),
$$

where $\frac{\mathrm{d} x_{\mathrm{st}}}{\mathrm{d} t}, \frac{\mathrm{d} y_{\mathrm{st}}}{\mathrm{d} t}$ are the usual total derivatives and $\frac{1}{m}$ is a scale factor with $m$ as given in (2.6). When we now consider the momentum equations in the stereographic $x_{\mathrm{st}}$ - and $y_{\mathrm{st}}$-direction, the 


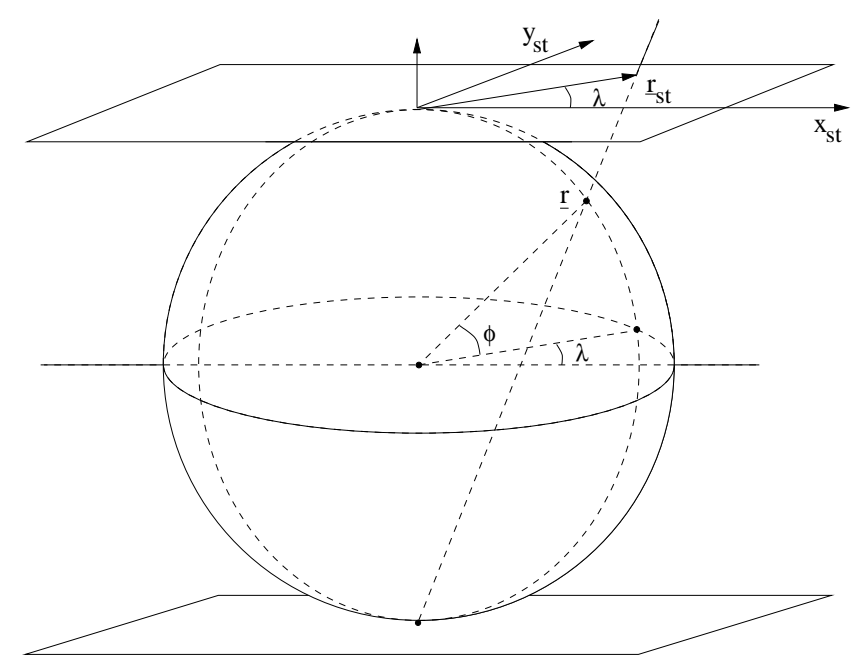

Figure 1: The stereographic planes for the northern and southern hemisphere projections.

stereographic formulation of the SWEs in flux-form reads

$$
\begin{aligned}
\frac{\partial H}{\partial t}+\nabla \cdot(H \underline{U}) & =0 \\
\frac{\partial H U}{\partial t}+\nabla \cdot(H U \underline{U}) & =\left[\alpha f-\frac{\left(x_{\mathrm{st}} V-y_{\mathrm{st}} U\right)}{2 a^{2}}\right] H V-m g H \frac{\partial h}{\partial x_{\mathrm{st}}}, \\
\frac{\partial H V}{\partial t}+\nabla \cdot(H V \underline{U}) & =-\left[\alpha f-\frac{\left(x_{\mathrm{st}} V-y_{\mathrm{st}} U\right)}{2 a^{2}}\right] H U-m g H \frac{\partial h}{\partial y_{\mathrm{st}}},
\end{aligned}
$$

where the divergence operator is defined by

$$
\nabla \cdot(A \underline{U}) \equiv m^{2} \frac{\partial}{\partial x_{\mathrm{st}}}\left(\frac{A U}{m}\right)+m^{2} \frac{\partial}{\partial y_{\mathrm{st}}}\left(\frac{A V}{m}\right) .
$$

This formulation is derived in Appendix B.

To complete the discussion on the two different coordinate systems, we here give the relations between the stereographic and spherical velocity components,

$$
\begin{aligned}
U & =-u \sin \lambda-\alpha v \cos \lambda \\
V & =u \cos \lambda-\alpha v \sin \lambda .
\end{aligned}
$$

These relations, which of course are only valid outside the poles, are needed in Section 3.2.4.

\section{Spatial Discretization}

\subsection{Using stereographic grids}

Over the years several suggestions have been made to circumvent the singularity problem which arises at the poles when one tries to solve the SWEs in spherical coordinates. Already in 1956 Phillips [17] studied this problem. He suggested to cover the sphere with three different coordinate systems. On part of the northern as well as on the southern hemisphere he used a stereographic coordinate system centered at the poles. In between those two regions he chose a mercator projection. His distribution of the coordinate systems is illustrated in Figure 2(a). To couple the different coordinate systems Phillips had to interpolate from points in neighbouring grids, whenever a variable outside the current grid part was needed. In 1975 Stoker [21] showed that these interpolations could contribute to loss of mass.

In 1977 Starius [20] introduced the composite mesh method. Like Phillips, he also used multiple coordinate systems, but he avoided interpolations within neighbouring grids by letting the grids, 
corresponding with the different coordinate systems, overlap. To prosper from both methods Browning [2] combined the ideas of Starius and Phillips. He applied the composite mesh method to the SWEs by using two stereographic coordinate systems which he centered respectively at the north and south pole and extended beyond the equator.

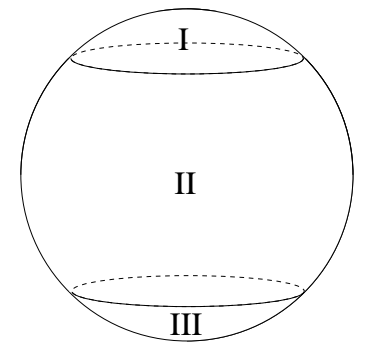

(a) (I) northern hemisphere stereographic projection (II) mercator projection

(III) southern hemisphere stereographic projection

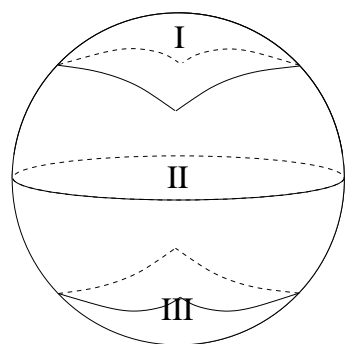

(b) (I) northern hemisphere stereographic projection (II) spherical coordinate system (III) southern hemisphere stereographic projection

Figure 2: Distribution of the three different coordinate systems in Phillips' approach (a) and our approach (b).

Our approach is also based on the ideas of Phillips, that is, we use three different non-overlapping coordinate systems, where stereographic coordinate systems are applied in the northern and southern polar areas. In the intermediate region however, our choice of the coordinate system differs from Phillips'. Since spherical coordinates are natural and easily implemented in regions away from the poles, we prefer a spherical coordinate system in the intermediate region. Furthermore, latlon grids are still standard in meteorological applications. A further differentiation from Phillips' method concerns the coupling of the different coordinate systems. Although this subject is not addressed until Section 3.2, we state here that with our choice of a finite volume method we are able to avoid the interpolation problems as found in Phillips. Our distribution of the coordinate systems is shown in Figure 2(b).

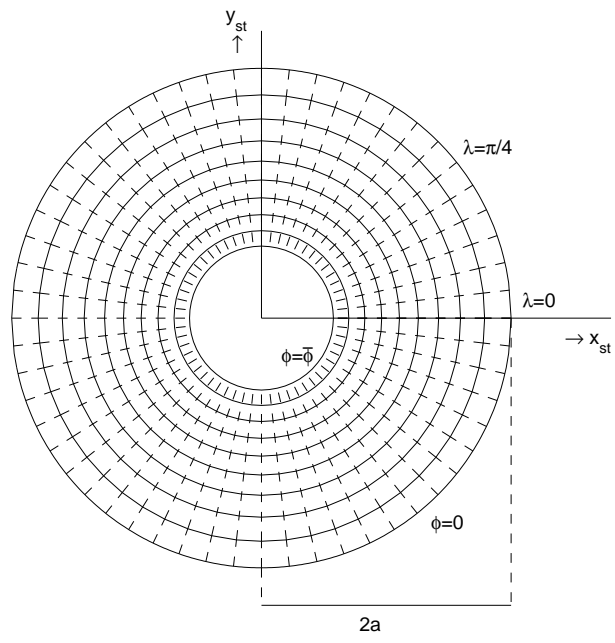

Figure 3: Northern hemisphere projection from the south pole of a uniform lat-lon grid. Dashed lines correspond with meridians $(\lambda$ constant). Solid lines correspond with parallels $(\phi$ constant). 
In this paragraph we discuss the exact distribution of the three different coordinate systems across the sphere. As mentioned before, we prefer to use a lat-lon grid in a region away from the poles. We define this region as $R_{I I}=\left\{(\lambda, \phi, a): \lambda \in[0,2 \pi), \phi \in[-\bar{\phi}, \bar{\phi}]\right.$ with $\left.\bar{\phi}<\frac{\pi}{2}\right\}$. From an illustrative point of view we assume that our lat-lon grid has a uniform distribution. Note that more advanced grid distributions are possible. In Section 4 for instance we apply a reduced lat-lon grid. To find a suitable grid distribution in the stereographic regions, we project the uniform latlon grid of region $R_{I I}$ onto the stereographic planes as illustrated in Figure 3. Note that meridians and parallels correspond with respectively dashed and solid lines. In the middle of the resulting projection we place a square with bottom left-hand corner $\left(x_{\mathrm{st}}, y_{\mathrm{st}}\right)=\left(-x_{r},-x_{r}\right)$ and top righthand corner $\left(x_{\mathrm{st}}, y_{\mathrm{st}}\right)=\left(x_{r}, x_{r}\right), x_{r}>0$. The corresponding regions on the sphere are denoted by region I and III. To secure a proper fit between the grids on region I, III and region $R_{I I}$, we extend the projected meridians, until they intersect with the square. Region $R_{I I}$ so expands to region II shown in Figure 2(b). We then demand that $N_{\lambda}$ defined as $N_{\lambda}=\frac{1}{\Delta \lambda}$ is a multiple of eight. Under this condition the intersection points have mirror images on the opposite edge. After connecting these points, a non-uniform rectangular grid distribution can be found on the square, see Figure 4(a). The total grid distribution over the sphere is now fully known, see Figure 4(b). Finally, we remark that $x_{r}, N_{\lambda}$ and $\bar{\phi}$ are still free parameters. Exact values are given for each test case. Their choices influence, for instance, the CFL-number, the meshwidth factors, and the accuracy. For visualization purposes we here used $N_{\lambda}=56, x_{r}=0.32279$ and $\bar{\phi}=57.8^{\circ}$.

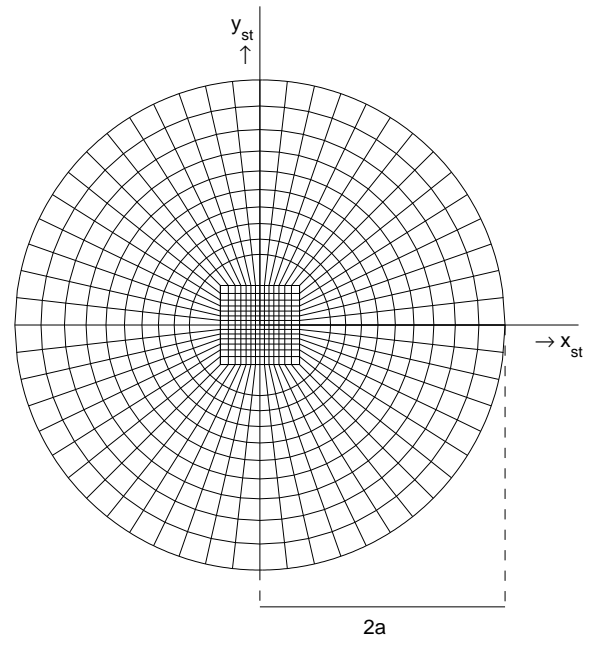

(a) Northern hemisphere stereographic projection of the grid.

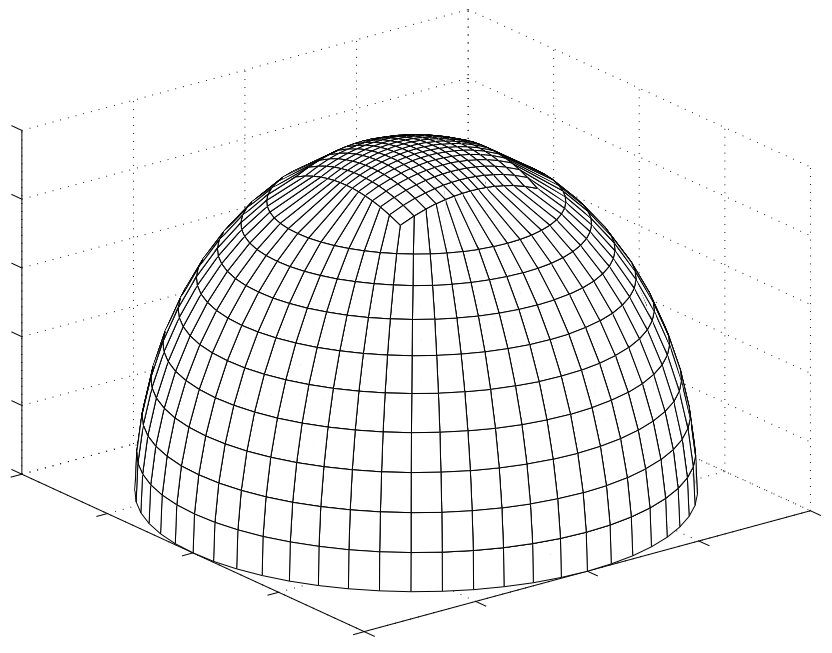

(b) Side view of the grid over the northern hemisphere.

Figure 4: Different views of the grid distribution over the northern hemisphere.

\subsection{The semi-discrete system in general terms}

Without the Coriolis and additional forces, the SWEs closely resemble the Euler equations, which can be found in, for instance, gas dynamic applications. For the full set of primitive equations this resemblance is even more explicit. A lot of theory concerning the space discretization of the Euler equations has already been developed, see for instance [11]. In our approximation method we gratefully adopt existing ideas from this theory. In this section we will describe the semi-discrete system for the SWEs (2.1)-(2.3) and (2.7)-(2.9) with special attention to the coupling between the spherical and stereographic grids.

3.2.1 Main outline of the finite volume method We begin this section with a main outline of our method. To guarantee conservation of mass and momentum in our semi-discrete system, or 
in other words to fully respect the underlying physical conservation laws, we use the finite volume method, which is standard practice for the Euler equations. We focus on the stereographic region I. Similar results can be derived for the spherical region II and for region III. Calculations are done in the computational domain, which results after projection of the regions I, II, and III on the regions associated with the corresponding coordinate systems. In the computational domains regular (non-)uniform rectangular grids occur.

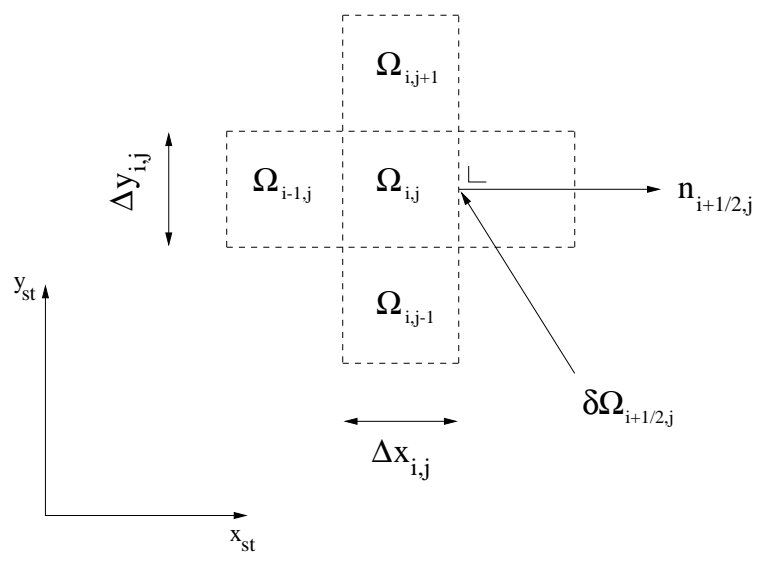

Figure 5: The grid cell $\Omega_{i, j}$ in the stereographic coordinate system.

Let $\Omega_{i, j}$ be a grid cell with boundary $\delta \Omega_{i, j}$. We denote its four neighbours by $\Omega_{i \pm 1, j}$ and $\Omega_{i, j \pm 1}$. The boundary between two neighbouring cells, for instance, between $\Omega_{i+1, j}$ and $\Omega_{i, j}$ is denoted by $\delta \Omega_{i+1 / 2, j} \cdot \underline{n}_{i+1 / 2, j}=\left(n_{x_{\mathrm{st}}}, n_{y_{\mathrm{st}}}\right)$ is the outwardly directed unit normal along this boundary. $\Delta x_{i, j}$ and $\Delta y_{i, j}$ are respectively the lengths of $\delta \Omega_{i, j \pm 1 / 2}$ and $\delta \Omega_{i \pm 1 / 2, j}$, see Figure 5 . We associate with each grid cell its cell center ${\underline{x_{\mathrm{st}}}}_{i, j}=\left(x_{\mathrm{st}_{i, j}}, y_{\mathrm{st}_{i, j}}\right)$ with state variable $\underline{q}_{i, j}=\left(H_{i, j}, H_{i, j} U_{i, j}, H_{i, j} V_{i, j}\right)$ and we assume that the state variable is constant over each cell. The finite volume method now gives

$\frac{\partial \underline{q}_{i, j}}{\partial t}+\frac{m_{i, j}^{2}}{\Delta x_{i, j} \Delta y_{i, j}} \oint_{\delta \Omega_{i, j}} \frac{1}{m} \underline{F} n_{x_{\mathrm{st}}}+\frac{1}{m} \underline{G} n_{y_{\mathrm{st}}} d S=-\left(\underline{f}_{x_{\mathrm{st}}}\left(\underline{q}_{i, j}, \underline{x}_{\mathrm{st}}{ }_{i, j}\right)+\underline{f}_{y_{\mathrm{st}}}\left(\underline{q}_{i, j},{\underline{x_{\mathrm{st}}}}_{i, j}\right)\right)$,

where $\underline{F}$ and $\underline{G}$ are the fluxes in stereographic $x_{\mathrm{st}^{-}}$and $y_{\mathrm{st}}-$ direction,

$$
\begin{aligned}
& \underline{F}(\underline{q})=\left(H U, H U^{2}+\frac{1}{2} g H^{2}, H U V\right)^{T}, \\
& \underline{G}(\underline{q})=\left(H V, H U V, H V^{2}+\frac{1}{2} g H^{2}\right)^{T},
\end{aligned}
$$

and

$$
\begin{aligned}
& \underline{f}_{x_{\mathrm{st}}}\left(\underline{q}, \underline{x_{\mathrm{st}}}\right)=\left(0,-\left[\alpha f-\frac{\left(x_{\mathrm{st}} V-y_{\mathrm{st}} U\right)}{2 a^{2}}\right] H V+m g H \frac{\partial h_{s}}{\partial x_{\mathrm{st}}}+\frac{1}{4 a^{2}} g H^{2} x_{\mathrm{st}}, 0\right)^{T}, \\
& \underline{f}_{y_{\mathrm{st}}}\left(\underline{q}, \underline{x_{\mathrm{st}}}\right)=\left(0,0,\left[\alpha f-\frac{\left(x_{\mathrm{st}} V-y_{\mathrm{st}} U\right)}{2 a^{2}}\right] H U+m g H \frac{\partial h_{s}}{\partial y_{\mathrm{st}}}+\frac{1}{4 a^{2}} g H^{2} y_{\mathrm{st}}\right)^{T} .
\end{aligned}
$$

To respect the characteristic directions associated with the hyperbolic character of our equations, we apply an upwind scheme to discretize the integral in (3.1). Within the group of finite volume upwind methods we distinguish two different categories. It concerns flux vector splitting (FVS) and flux difference splitting (FDS) methods. For a detailed description of both methods we refer to [11]. Methods from the first category do not suffice as discretization scheme when applied to 
the SWEs. The condition that the Jacobian of the flux vector $\underline{F}$ with respect to $\underline{q}$ is homogeneous of degree one, see [11], is not fulfilled. We apply an Osher approximate Riemann solver [15, 16], which makes an excellent choice from the group of FDS methods. Osher's scheme is robust and higher order accurate, when combined with the right state interpolation [22]. Furthermore, from a future perspective, it has a logical extension to more realistic primitive equations and a nice boundary treatment. The last argument made us decide in favor of Osher's approximate Riemann solver before Roe's one, which is often used in gasdynamical practice.

The semi-discrete system reads

$$
\begin{aligned}
& \frac{\partial \underline{q}_{i, j}}{\partial t}+\frac{m_{i, j}^{2}}{\Delta x_{i, j} \Delta y_{i, j}}\left[T^{-1}(0) \underline{F}_{(\mathrm{O})}\left(T(0) \underline{q}_{i+\frac{1}{2}, j}^{\mathrm{L}}, T(0) \underline{q}_{i+\frac{1}{2}, j}^{\mathrm{R}}\right) \frac{\Delta y_{i, j}}{m_{i+\frac{1}{2}, j}}+\right. \\
& T^{-1}\left(\frac{\pi}{2}\right) \underline{F}_{(\mathrm{O})}\left(T\left(\frac{\pi}{2}\right) \underline{q}_{i, j+\frac{1}{2}}^{\mathrm{L}}, T\left(\frac{\pi}{2}\right) \underline{q}_{i, j+\frac{1}{2}}^{\mathrm{R}}\right) \frac{\Delta x_{i, j}}{m_{i, j+\frac{1}{2}}}+ \\
& T^{-1}(\pi) \underline{F}_{(\mathrm{O})}\left(T(\pi) \underline{q}_{i-\frac{1}{2}, j}^{\mathrm{L}}, T(\pi) \underline{q}_{i-\frac{1}{2}, j}^{\mathrm{R}}\right) \frac{\Delta y_{i, j}}{m_{i-\frac{1}{2}, j}}+ \\
& \left.T^{-1}\left(\frac{3 \pi}{2}\right) \underline{F}_{(\mathrm{O})}\left(T\left(\frac{3 \pi}{2}\right) \underline{q}_{i, j-\frac{1}{2}}^{\mathrm{L}}, T\left(\frac{3 \pi}{2}\right) \underline{q}_{i, j-\frac{1}{2}}^{\mathrm{R}}\right) \frac{\Delta x_{i, j}}{m_{i, j-\frac{1}{2}}}\right] \\
& =-\left(\underline{f}_{x_{\mathrm{st}}}\left(\underline{q}_{i, j}, \underline{x}_{\mathrm{st}} i, j\right)+\underline{f}_{y_{\mathrm{st}}}\left(\underline{q}_{i, j}, \underline{x}_{\mathrm{st}} i, j\right)\right),
\end{aligned}
$$

where $T(\theta)$ is a rotation matrix defined by

$$
T(\theta)=\left(\begin{array}{ccc}
1 & 0 & 0 \\
0 & \cos \theta & \sin \theta \\
0 & -\sin \theta & \cos \theta
\end{array}\right)
$$

and $\underline{F}_{(\mathrm{O})}$ is the Osher flux given as

$$
\underline{F}_{(\mathrm{O})}\left(\underline{q}^{\mathrm{L}}, \underline{q}^{\mathrm{R}}\right)=\frac{1}{2}\left(\underline{F}\left(\underline{q}^{\mathrm{L}}\right)+\underline{F}\left(\underline{q}^{\mathrm{R}}\right)\right)-\frac{1}{2} \int_{\underline{q}^{\mathrm{L}}}^{\underline{q}^{\mathrm{R}}}|A(\underline{q})| d \underline{q} .
$$

$A$ is here defined as the Jacobian of the fluxvector $\underline{F}$ with respect to $\underline{q}, A=\partial \underline{F} / \partial \underline{q}$. The absolute value of this Jacobian is defined by

$$
|A(\underline{q})|=P(\underline{q})|\Lambda| P^{-1}(\underline{q}),
$$

where $P$ and $\Lambda$ result from diagonalizing the Jacobian matrix as $A=P \Lambda P^{-1}$. Note that the Osher fluxes in (3.2) describe local fluxes, i.e., they point in the direction of the outwardly directed unit normal on the corresponding boundary. The Osher flux (3.4) approximates the local flux across a boundary $\delta \Omega$, which results when at the left and the right of this boundary the constant states $\underline{q}^{\mathrm{L}}$ and $\underline{q}^{\mathrm{R}}$ are found.

So far, we have not mentioned the evaluation of the constant states. It is through these evaluations that we are able to properly couple the different grids. Furthermore, the state evaluations determine the accuracy of our scheme. We attend to this topic in the next section. Remains to say that the Osher solver is special, because of the choice for the integration path in its flux (3.4). With the right choice, calculation of the Osher flux boils down to a maximum of five flux evaluations $\underline{F}(q)$. We use the P-variant suggested by Hemker and Spekreijse [10]. Details of the construction of the integration path and the Osher flux can be found in Appendix C.

3.2.2 Determination of the constant states In this section we define the constant states. We still focus on the stereographic region zooming in on the state evaluation in the $x_{\text {st }}$-direction. The states in the $y_{\mathrm{st}}$-direction are defined in a similar way. We apply $1 \mathrm{D}$ state interpolation, i.e., the state $\underline{q}_{i+\frac{1}{2}, j}^{\mathrm{L}}$ only depends on the states of neighbouring cells in the $x_{\mathrm{st}}$-direction. For the 
remaining part of this subsection, we suppress the index $j$ in our notation. To define the constant states, we use the $(\kappa=1 / 3)$-scheme [22]. On a uniform grid it reads

$$
\begin{aligned}
& \underline{q}_{i+\frac{1}{2}}^{\mathrm{L}}=\underline{q}_{i}+\frac{(1-\kappa)}{4}\left(\underline{q}_{i}-\underline{q}_{i-1}\right)+\frac{(1+\kappa)}{4}\left(\underline{q}_{i+1}-\underline{q}_{i}\right), \\
& \underline{q}_{i+\frac{1}{2}}^{\mathrm{R}}=\underline{q}_{i+1}+\frac{(1-\kappa)}{4}\left(\underline{q}_{i+1}-\underline{q}_{i+2}\right)+\frac{(1+\kappa)}{4}\left(\underline{q}_{i}-\underline{q}_{i+1}\right) .
\end{aligned}
$$

Unfortunately, our grid in the projected stereographic region is non-uniform. When the grid is sufficiently smooth, this discrepancy is often circumvented by simply applying the existing $\kappa$ scheme (3.5). To avoid unnecessary irregulations, so to get a clear view of possible problems arising due to the coupling between the different grids, we have decided to modify the $\kappa$-scheme for non-uniform grids. In Appendix D the general form of this modified $\kappa$-scheme is given for different values of $\kappa$. The general form is defined as a function $I_{\kappa}$ with the states and cell widths of neighbouring grid cells in the interpolation direction as arguments. The standard non-uniform state interpolation is represented in Table 1.

Near the grid interface between the stereographic and spherical region, see Figure 4(a), the stencil of the non-uniform $(\kappa=1 / 3)$-scheme is too large, demanding state variables from outside the stereographic region. To avoid transformations and difficulties associated with the kink in the grid cells, we regard the grid interface as a real boundary. This means that we locally have to reduce the size of our stencil. To that end we have also formulated the non-uniform equivalents of the 2-point central $(\kappa=1)$-scheme, the 2-point upwind $(\kappa=-1)$-scheme, and the 3-point upwind $(\kappa=1 / 2)$-scheme. Figure 6 shows which interpolation scheme is applied on which cell boundary. The associated state interpolations are given in Table 1 . Note that although it is a 3 -point interpolation scheme, the $(\kappa=1 / 3)$-scheme, as opposed to the $(\kappa=1 / 2)$-scheme, can not be applied at the cell boundaries $\delta \Omega_{5 / 2}$ and $\delta \Omega_{N-3 / 2}$, because in these cases a cell width from outside the stereographic region is needed. In the next section, we will discuss the special Transformation entry in Table 1.

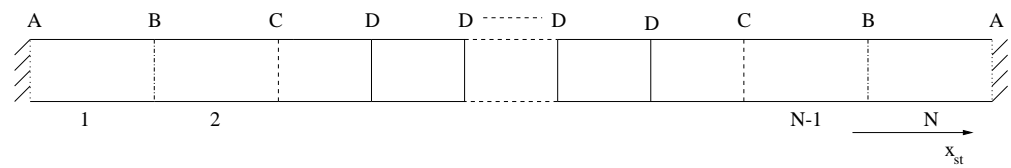

Figure 6: Illustration of the cell boundaries, where another interpolation scheme than standard is needed.

3.2.3 The finite volume method and the constant states on the spherical computational domain. The same line of semi-discretization as described in Section 3.2.1 is applied to derive the semidiscrete system for the region II, see Figure 2(b). Note that for this region calculations are done on the $(\lambda, \phi)$-plane. The semi-discrete system easily follows through equations (3.2)-(3.4), when we replace $m_{i, j}, \Delta x_{i, j}, \Delta y_{i, j}, \underline{f}_{x_{\mathrm{st}}}, \underline{f}_{y_{\mathrm{st}}}$ and $\underline{q}$ successively by $1 /\left(a \cos \phi_{i, j}\right), \Delta \lambda_{i, j}, \Delta \phi_{i, j}, \underline{f}_{\lambda}, \underline{f}_{\phi}$ and $\underline{q}=(H, H u, H v)$, where

$$
\begin{aligned}
& \underline{f}_{\lambda}(\underline{q}, \underline{r})=\left(0,-\left(f+\frac{u}{a} \tan \phi\right) H v+\frac{g H}{a \cos \phi} \frac{\partial h_{s}}{\partial \lambda}, 0\right)^{T}, \\
& \underline{f}_{\phi}(\underline{q}, \underline{r})=\left(0,0,\left(f+\frac{u}{a} \tan \phi\right) H u+\frac{g H^{2} \sin \phi}{2 a \cos \phi}+\frac{g H}{a} \frac{\partial h_{s}}{\partial \phi}\right)^{T} .
\end{aligned}
$$

Note that the form of the flux vectors $\underline{F}$ and $\underline{G}$ remains the same, since both coordinate systems are conformal.

To evaluate the constant states on region II we again use 1D state interpolation. This time it concerns interpolation in the $\lambda$ - or $\phi$-direction depending on the cell boundary under consideration. As standard interpolation scheme the $(\kappa=1 / 3)$-scheme is applied. In the $\lambda$-direction this 


\begin{tabular}{|c|c|c|}
\hline & Left & Right \\
\hline $\mathrm{A}$ & $\begin{array}{l}\underline{q}_{\frac{1}{2}}^{\mathrm{L}}=\text { Transformation } \\
\underline{q}_{\frac{1}{2}}^{\mathrm{R}}=I_{-1}\left(\underline{q}_{2}, \underline{q}_{1}, \ell_{2}, \ell_{1}\right)\end{array}$ & $\begin{array}{l}\underline{q}_{N+\frac{1}{2}}^{\mathrm{L}}=I_{-1}\left(\underline{q}_{N-1}, \underline{q}_{N}, \ell_{N-1}, \ell_{N}\right) \\
\underline{q}_{N+\frac{1}{2}}^{\mathrm{R}}=\text { Transformation }\end{array}$ \\
\hline B & $\begin{array}{l}\underline{q}_{\frac{3}{2}}^{\mathrm{L}}=I_{1}\left(\underline{q}_{1}, \underline{q}_{2}, \ell_{1}, \ell_{2}\right) \\
\underline{q}_{\frac{3}{2}}^{\mathrm{R}}=I_{\frac{1}{2}}\left(\underline{q}_{3}, \underline{q}_{2}, \underline{q}_{1}, \ell_{3}, \ell_{2}, \ell_{1}\right)\end{array}$ & $\begin{array}{l}\underline{q}_{N-\frac{1}{2}}^{\mathrm{L}}=I_{\frac{1}{2}}\left(\underline{q}_{N-2}, \underline{q}_{N-1}, \underline{q}_{N}, \ell_{N-2}, \ell_{N-1}, \ell_{N}\right) \\
\underline{q}_{N-\frac{1}{2}}^{\mathrm{R}}=I_{1}\left(\underline{q}_{N}, \underline{q}_{N-1}, \ell_{N}, \ell_{N-1}\right)\end{array}$ \\
\hline $\mathrm{C}$ & $\underline{q}_{\frac{5}{2}}^{\mathrm{L}}=I_{\frac{1}{2}}\left(\underline{q}_{1}, \underline{q}_{2}, \underline{q}_{3}, \ell_{1}, \ell_{2}, \ell_{3}\right)$ & $\underline{q}_{N-\frac{3}{2}}^{\mathrm{R}}=I_{\frac{1}{2}}\left(\underline{q}_{N}, \underline{q}_{N-1}, \underline{q}_{N-2}, \ell_{N}, \ell_{N-1}, \ell_{N-2}\right)$ \\
\hline $\mathrm{D}$ & \multicolumn{2}{|c|}{$\begin{array}{l}\underline{q}_{i+\frac{1}{2}}^{\mathrm{L}}=I_{\frac{1}{3}}\left(\underline{q}_{i-1}, \underline{q}_{i}, \underline{q}_{i+1}, \ell_{i-2}, \ell_{i-1}, \ell_{i}, \ell_{i+1}, \ell_{i+2}\right) \\
\underline{q}_{i+\frac{1}{2}}^{\mathrm{R}}=I_{\frac{1}{3}}\left(\underline{q}_{i+2}, \underline{q}_{i+1}, \underline{q}_{i}, \ell_{i+3}, \ell_{i+2}, \ell_{i+1}, \ell_{i}, \ell_{i-1}\right)\end{array}$} \\
\hline
\end{tabular}

Table 1: The different state interpolation methods used near the grid boundary. The indices A, $\mathrm{B}, \mathrm{C}$ and $\mathrm{D}$ here correspond with the different cell boundary situations illustrated in Figure 6 .

scheme can be applied everywhere, because, in that direction, our grid is uniform and has no grid boundaries. In the $\phi$-direction we have to account for the grid interface between the spherical and the stereographic grids. We treat this interface as if it concerns a piecewise constant real boundary approximating the cell boundaries by the lines $\phi=\phi_{i, N_{\phi}+1 / 2}$, see Figure 7. The resulting partially non-uniform grid distribution resembles the one in the stereographic direction. Therefore, the associated state interpolations easily follow by applying Table 1 in the $\phi$-direction.

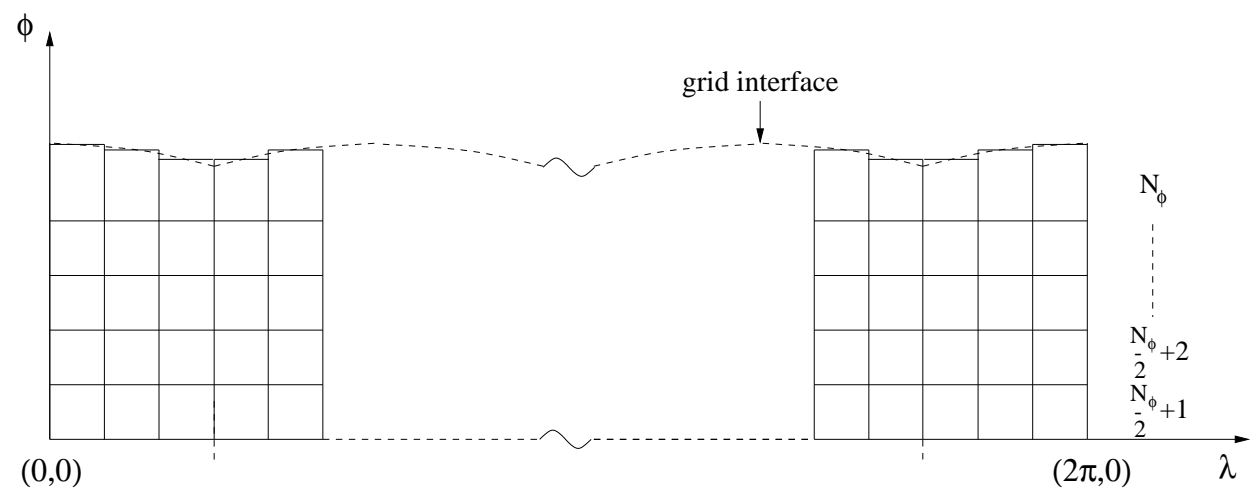

Figure 7: Projection of the northern hemisphere part of region II on the $(\lambda, \phi)$-plane in combination with the approximated cell distribution at the grid boundary.

3.2.4 Interaction between the different computational domains. Remains to discuss the Transformation entry in Table 1. We again turn to the stereographic computational domain associated with region I and focus on the $x_{\mathrm{st}}$-direction, see Figure 6 . At the grid interface between region I and II the computational domains of these regions interact. To find the states $\underline{q}_{1 / 2}^{\mathrm{L}}$ and $\underline{q}_{N+1 / 2}^{\mathrm{R}}$ in stereographic variables, we transform the states in spherical variables found at the same cell interface boundary in the computational domain associated with region II. The word transform here indicates that we must convert the velocity field $\underline{u}=(u, v)$ into its stereographic representation. Note that the constant states in spherical variables are calculated by one-sided $(\kappa=-1)$-state 
interpolation in the $\phi$-direction. This way of state evaluation yields that at every cell interface boundary, the $1 \mathrm{D}$ state interpolation to obtain $\underline{q}_{1 / 2}^{\mathrm{L}}$ and $\underline{q}_{N+1 / 2}^{\mathrm{R}}$ is performed in a different direction, i.e. in the direction of the projected meridians $\lambda_{1 / 2}$ and $\lambda_{N+1 / 2}$. Though simple, this choice of varying interpolation directions influences our approximated solution to the SWEs. In case of interpolations in the $\phi$-direction, the Transformation entries, i.e. $\underline{q}_{1 / 2}^{\mathrm{L}}$ and $\underline{q}_{N_{\phi}+1 / 2}^{\mathrm{R}}$ in spherical variables, follow after transforming the corresponding constant states in stereographic variables found at the same cell boundaries in the computational domain of region I. Here the word "Transformation" means that we must convert the velocity field $\underline{U}=(U, V)$ into its spherical equivalent. Note that, depending on the cell's position, the constant state in stereographic variables concerns a constant state calculated by one-sided $(\kappa=-1)$-state interpolation in $x_{\text {st }}$ - or $y_{\text {st }}$-direction.

We conclude this section with some remarks on accuracy. In more dimensional problems a finite volume method is at most second order accurate. To provide an order estimate we cite Spekreijse [18]. For a uniform grid, he proved: A scheme like (3.2) is second order accurate for interpolations based on the $\kappa$-scheme. On a large part of our domain, i.e. almost everywhere on the spherical region, see Section 3.2.3, we can use his estimate, because our grid is uniform. However, since we combine different grids, it is difficult to give the exact order of our scheme across the whole sphere. It is obvious that we endure some accuracy loss around the interface, which will be referred to as the connection problem. To be conclusive about its severity, we will give a numerical order estimate in Section 4.

\section{NumERICAL TESTS}

In this section we focus on two main objectives. First, we wish to establish to what extent the introduction of the stereographic grid resolves the problems related to the use of a global spherical coordinate system. Second, we are interested in the performance of our spatial discretization scheme. Or in other words, how well does Osher's scheme perform, when applied to the SWEs on the sphere, and how accurate are its results.

To meet the necessity of a good benchmark to test new numerical methods to solve the SWEs in spherical geometry, Williamson et al. [26] developed a test set, containing seven different test cases of increasing complexity. We concentrate on test case two of this test set, i.e. on the global steady state non-linear zonal geostrophic flow. Test case two provides us with a good test to examine the scheme's ability to handle the poles. Furthermore, it serves as a test for our Osher scheme, since it includes non-linearity aspects of the SWEs. We also successfully solved test cases one and six, i.e. advection of a cosine bell over the pole and the Rossby-Haurwitz wave. To save space we only present results for test case two. In future work we will attend to the other cases.

\subsection{Test case two: Global steady state non-linear zonal geostrophic flow}

Test case two concerns a steady state analytic solution to the non-linear SWEs. It consists of a solid body rotation with the corresponding geostrophic height field $H$. A parameter $\alpha$ is used to specify the angle between the axis of the solid body rotation and the polar axis of the spherical coordinate system: $\alpha=0$ indicates equatorial flow and $\alpha=\pi / 2$ yields flow across the pole. The analytic solution of test case two reads

$$
\begin{aligned}
H & =h_{0}-\left(\frac{a \Omega u_{0}}{g}+\frac{u_{0}^{2}}{2 g}\right)(-\cos \lambda \cos \phi \sin \alpha+\sin \phi \cos \alpha)^{2}, \\
u & =u_{0}(\cos \phi \cos \alpha+\sin \phi \cos \lambda \sin \alpha) \\
v & =-u_{0} \sin \lambda \sin \alpha
\end{aligned}
$$

where the Coriolis parameter $f=2 \Omega(-\cos \lambda \cos \phi \sin \alpha+\sin \phi \cos \alpha)$ and $u_{0}=38.61 \mathrm{~m} / \mathrm{s}, h_{0}=$ $3.00 \cdot 10^{3} \mathrm{~m}$. To be consistent with the article of Williamson et al. [26], we tested our code for $\alpha=0,0.05, \pi / 2-0.05$, and $\pi / 2$, where the second and third parameter values were added to avoid symmetries. In this article we will not represent all the results as our code produced good results for either value. We will concentrate on tests with parameter value $\alpha=\pi / 2$, since for these tests the corresponding velocity components initiate the strongest flow across the poles. We remark that these kind of flows can indeed be encountered in practical situations. 
Besides the fact that we encounter a singularity problem when we apply the spherical formulation of the SWEs in the poles, we already have to deal with some additional problems when approaching the poles. Probably the best way to stress the need for additional caution near the poles is to show Figure 8. This figure represents the analytic longitudinal and latitudinal velocity components, $u$ and $v$, found in the cell centers of an underlying uniform lat-lon grid in case of flow across the poles $(\alpha=\pi / 2)$. To emphasize our point we give the velocity components $u$ and $v$, which follow from (4.1)-(4.3)

$$
\begin{aligned}
& u=u_{0} \sin \phi \cos \lambda, \\
& v=-u_{0} \sin \lambda .
\end{aligned}
$$

The figure shows that the spherical velocity components strongly vary in the polar area, bringing about difficulties in numerical approximation methods. To properly represent these velocity components, a fine grid resolution, especially in the longitudinal direction, is necessary. However, too much grid cells can lead to problems for integration methods related to stability.
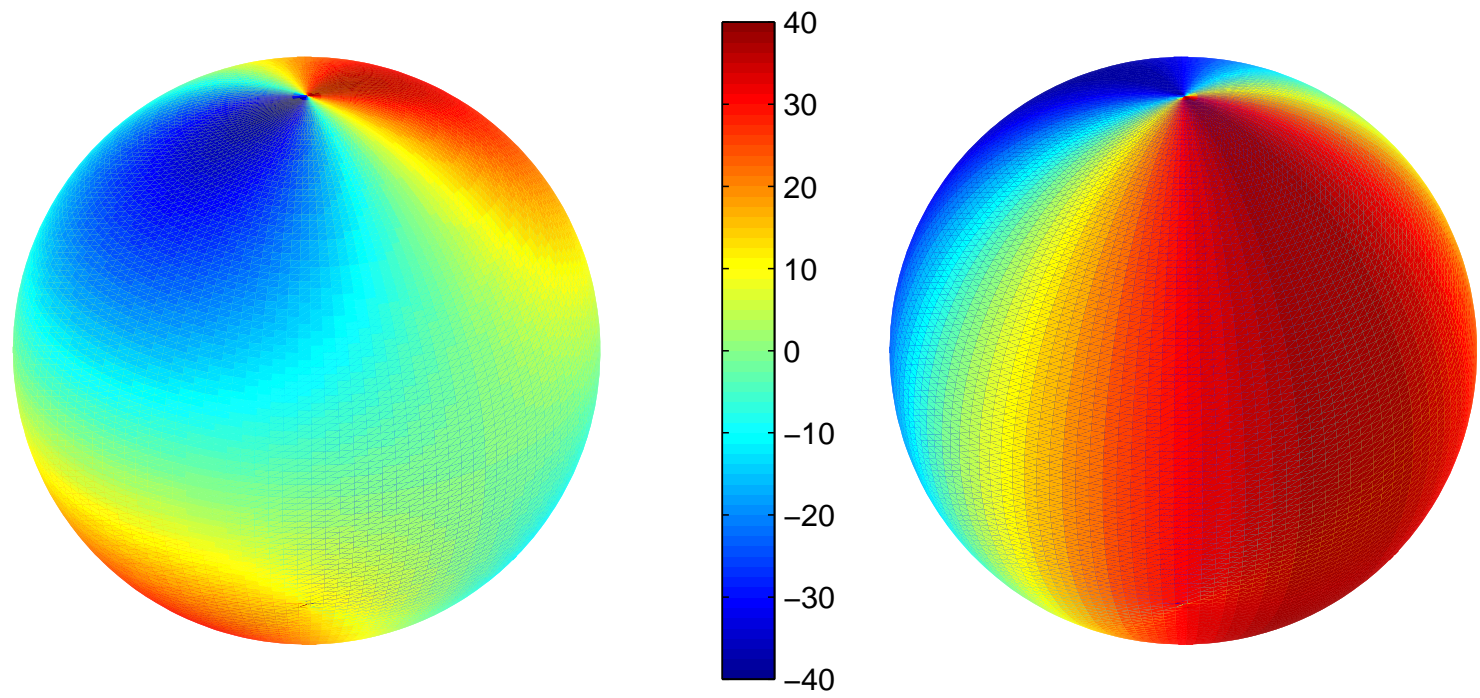

Figure 8: Representation of the analytic longitudinal velocity component $u$ (left) and latitudinal velocity component $v$ (right) on a global uniform lat-lon grid in case of global steadystate non-linear zonal geostrophic flow across the pole $(\alpha=\pi / 2)$.

We discuss two remedies to these approximation and stability problems. First, we can decide to solve the SWEs on a stereographic grid. On a stereographic grid no severe resolution problems arise, as the velocity components $\mathrm{U}$ and $\mathrm{V}$ vary much less than the spherical ones, see Figure 9 . Second, we can consider the reduced grid approach. In that case, the lat-lon grid is coarsened in the longitudinal direction at given latitudes. For details we direct to [1] and [24]. Both remedies suffer some problems though. On a (nearly) global lat-lon grid, we are not allowed to apply the reduced grid approach to its fullest extent. Repeated reductions to arrive for instance at four remaining grid cells next to the poles, are inadmissible, since in that case the grid near the poles is too coarse to represent the strongly varying velocity components. On a stereographic grid, we are confronted with a connection problem at the equator when we try to combine the stereographic grids on the northern and southern hemisphere, see Figure 9. With a combination of both remedies, i.e. a combined grid with a reduced lat-lon grid away from the poles and a stereographic grid at the two polar caps, we can avoid these problems and benefit from either advantages, see Figure 10.

In the remaining part of this section we will address the following questions concerning our grid. Do the numerical results confirm the problems suggested when calculating on a global reduced lat-lon grid? Which factors determine the actual form of a combined grid, or in other words, how 

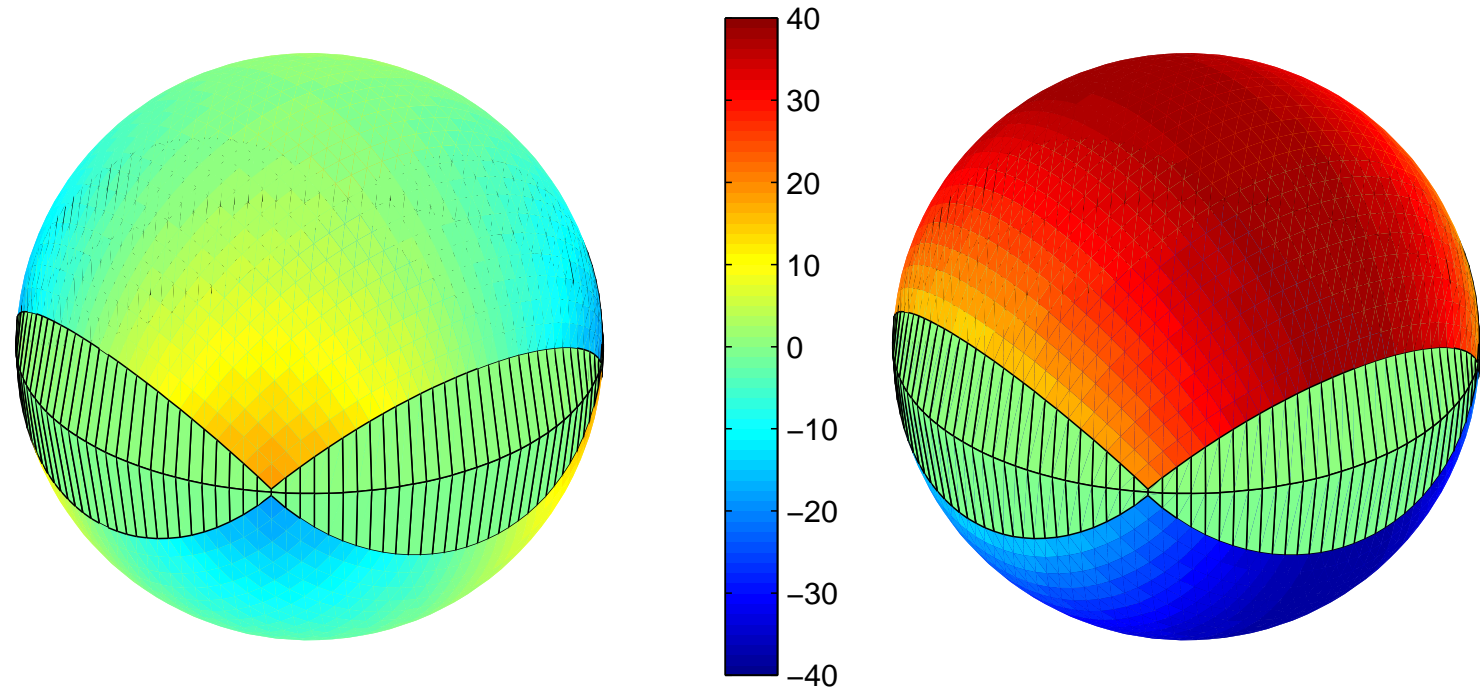

Figure 9: Representation of the analytic stereographic velocity components $U$ (left) and $V$ (right) on a "global" uniform stereographic grid in case of global steady state non-linear zonal geostrophic flow across the pole.

large should the stereocap be and how much reductions are allowed? And, how accurate are the results when calculated on a combined grid with realistic refinement?

\subsubsection{Experiments on global lat-lon grids.}

The pole singularity For tests on a global lat-lon grid to make sense, we have to account for the non-existence of the spherical fluxes $\underline{F}$ and $\underline{G}$ in the poles. In practice, this problem is overcome by assuming a total zero flux across the boundaries corresponding to the poles. The question is whether or not the results significantly suffer from this assumption, both near and away from the poles. In fact, when the results do suffer from this assumption, we should reconsider investigating the global reduced lat-lon grid, since the results would be inadequate without an accurate resolvement of the singularity problem in the pole.

We first ran a set of tests on a rectangular global lat-lon grid, where we varied the amount of gridpoints in the $\phi$-direction, thus moving the neighbouring cell centers closer to the pole with each test. Let $\mathrm{nP}$ define the amount of gridpoints in the $\phi$-direction and let $\Delta \phi=180^{\circ} / \mathrm{nP}$. In comparison with other tests, our grid distribution in the $\lambda$-direction is rather coarse $(\mathrm{nL}=72)$. Since we are not interested in the very accurate results, we only have to make sure that the solution can be properly represented in that direction. In this way we are able to reduce on computing time and avoid problems related to stability. The error measures on $H$ are shown in Table 2. For time stepping we used the 4-th order Runge-Kutta method with small steps, such that the error $E_{\mathrm{r}}(H)$ represents the spatial discretization error. $E_{\mathrm{r}}(H)$ is defined as a maximum relative error

$$
E_{\mathrm{r}}(H)=\max _{(i, j)}\left|\frac{H_{i, j}-H\left(\lambda_{i}, \phi_{j}\right)}{H\left(\lambda_{i}, \phi_{j}\right)}\right|,
$$

where $H\left(\lambda_{i}, \phi_{j}\right)$ gives the analytic solution of $H$ in cell center $(i, j)$. The max-norm is taken over a specified region. Note that since for $H$ holds $H \gg 1$, the relative error provides a good indication of the accuracy of our results.

Table 2 clearly shows that in the band next to the poles the zero flux assumption does not lead to an error increase when approaching the poles. We even observe a minor decrease and the (relative) error certainly is sufficiently small for practical purposes. Moreover, the error in the pole 


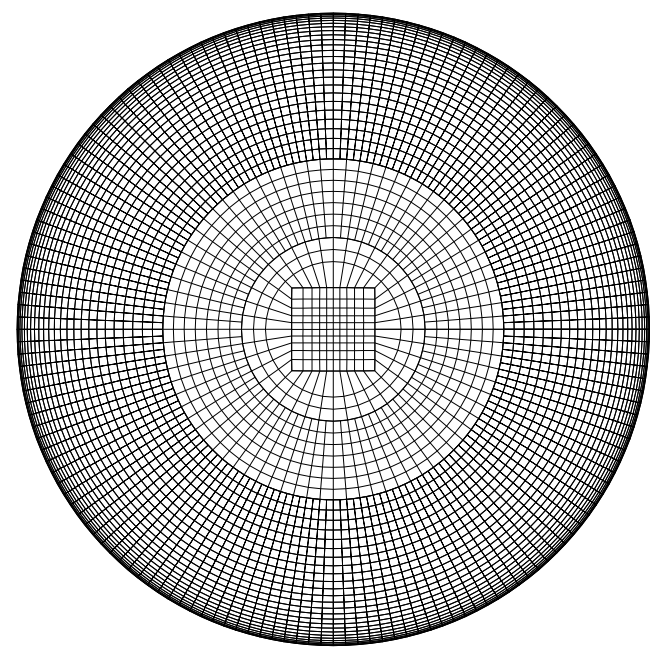

Figure 10: Projection of a combined grid consisting of a reduced lat-lon grid away from the poles and a stereographic grid at the two polar caps onto the cartesian $(\mathrm{x}, \mathrm{y})$-plane $(\mathrm{z}=0)$. Two reductions were applied.

\begin{tabular}{|c|c|c|}
\hline & $E_{\mathrm{r}}(H)_{\text {pole band }}$ & $E_{\mathrm{r}}(H)_{\text {whole }}$ \\
\hline $\mathrm{nP}=36$ & $2.1 \cdot 10^{-3}$ & $9.8 \cdot 10^{-3}$ \\
\hline $\mathrm{nP}=72$ & $1.1 \cdot 10^{-3}$ & $5.7 \cdot 10^{-3}$ \\
\hline $\mathrm{nP}=180$ & $8.7 \cdot 10^{-4}$ & $5.3 \cdot 10^{-3}$ \\
\hline
\end{tabular}

Table 2: Error measures on $H$ for different values of $\mathrm{nP}$ taken over the volumes located next to the poles and over the whole domain on a rectangular lat-lon grid $(\mathrm{nL}=72)$.

band is smaller than the error over the whole domain. Note that since $\mathrm{nL}$ is fixed, convergence of the Osher scheme is not examined in these tests.

Pole resolution problem As mentioned before and as discussed by Williamson and Browning in [25], we encounter representation problems when we try to approximate the spherical velocity components on a too coarse grid around the poles. The following tests have been chosen to show the severity of this problem. We tested four different reduced rectangular lat-lon grids, all having $\mathrm{nL}(0)=64$ grid cells in the longitudinal direction and $\mathrm{nP}=192$ cells in latitudinal direction. $\mathrm{nL}(0)$ is here defined as the amount of grid cells in the longitudinal direction on the unreduced grid part. When approaching the poles, we halve the amount of grid cells in the longitudinal direction, whenever the cell width in that direction projected onto the sphere, i.e. $a \cos \phi \Delta \lambda$, is reduced with a factor two following the last reduction. The specific values for $\mathrm{nL}(0)=64$ and $\mathrm{nP}=192$ are chosen such that we can arrive on a coarse grid within a few reductions and for each grid part, containing the same amount of grid cells in longitudinal direction, enough grid cells in latitudinal direction are guaranteed. Successively, we apply 1, 2, 3 or 4 reductions at the latitudes $\phi=60^{\circ}, 75.9375^{\circ}, 82.5^{\circ}$ and $86.25^{\circ}$. The errors are displayed in Table 3 . This time we concentrate on the absolute error $E_{\mathrm{a}}(u)$ found for the velocity component $u$ instead of for $H$, since this component suffers the most from the inadequacy to represent the flux on a coarse lat-lon grid. Furthermore, the absolute error is shown, because the velocity component may vanish in certain 
points of the globe, see (4.4) and (4.5). $E_{\mathrm{a}}(u)$ is defined as the maximum absolute error

$$
E_{\mathrm{a}}(u)=\max _{i, j}\left|u_{i, j}-u\left(\lambda_{i}, \phi_{j}\right)\right|,
$$

where $u\left(\lambda_{i}, \phi_{j}\right)$ represents the analytic velocity component $u$ in cell center $\left(\lambda_{i}, \phi_{j}\right)$. The maximum is taken over the whole grid, where the second column entry indicates on which grid part $m$ the maximum error is found. The index $m$ denotes the grid part found between the $|m|$-th and $|m|+1$ th reduction. We indicate the different grid parts at the northern hemisphere with positive values of $m$ and at the southern hemisphere with negative values of $m$.

\begin{tabular}{|l|c|c|}
\hline & $E_{\mathrm{a}}(u)$ & grid part $m$ \\
\hline 0 reductions, & 0.32 & 0 \\
\hline 1 reduction at $\phi=60^{\circ}$ & 1.03 & $-1 / 1$ \\
\hline 2 reductions resp. at $\phi=60^{\circ}, 75.9375^{\circ}$ & 3.67 & $-2 / 2$ \\
\hline 3 reductions resp. at $\phi=60^{\circ}, 75.9375^{\circ}, 82.5^{\circ}$ & 15.18 & $-3 / 3$ \\
\hline 4 reductions resp. at $\phi=60^{\circ}, 75.9375^{\circ}, 82.5^{\circ}, 86.25^{\circ}$ & 23.99 & $-4 / 4$ \\
\hline
\end{tabular}

Table 3: Error measures on $u$ taken over the whole domain on a global reduced lat-lon grid with different levels of reduction $(\mathrm{nL}(0)=64, \mathrm{nP}=192)$. The second column displays on which grid part $m$ the maximum error is located.

Realizing that the analytic longitudinal velocity component $u$ has a maximum of $38,61 \mathrm{~m} / \mathrm{s}$, the results speak for themselves. It is obvious that a significant number of cells next to the poles is needed to properly represent the velocity components. For example, in this case and starting from $\mathrm{nL}(0)=64$, two reductions giving 16 cells next to the poles, already result in a maximum relative error in the longitudinal velocity component $u$ of about $10 \%$. Note that the maximum errors are found in the grid part closest to the pole.

Order tests In this part we provide a numerical order estimate for our spatial discretization scheme. As described in Section 3.2.4, we expect to find second order accuracy on a uniform grid. To verify this we ran some tests on a global uniform lat-lon grid. We only performed calculations on a band between latitudes $\phi=-60^{\circ}$ and $\phi=60^{\circ}$ to avoid small steps related to stability. On the other areas of the sphere we prescribed the analytic solution. Note that in this way accuracy losses due to the zero flux assumption across the poles are circumvented. Successively, we applied a uniform lat-lon grid with $\mathrm{nL}=72,144,288$ and 576. Table 4 shows the relative error measures on $H$. We consider the max-norm over the band.

The order factor between two successive grids is given in the third column of Table 4 . In case of second order accuracy this factor should be four. For the higher orders observed, we have two possible explanations. First, the theoretical order estimate holds in the asymptotic case, i.e. when $\mathrm{nL}$ approaches infinity. The order factor between the grids with $\mathrm{nL}=576$ and $\mathrm{nL}=288$ already moves closer to four. Second, on the band between the latitudes $\phi=-60^{\circ}$ and $\phi=60^{\circ}$, the flow has a strongly one-dimensional character which coincides with the meridians. For a uniform grid Spekreijse [18] proved: A scheme like (3.2) is third order accurate for interpolations based on the $(\kappa=1 / 3)$-scheme in the 1D case. This might explain why on the coarser grids our order factors are close to eight. Note that the value 5.1 can then be attributed to the fact that on finer grids the volumes move closer to the boundary of the band, where the one-dimensional character of our flow diminishes.

In case of a non-uniform grid we have no analytic order estimate. Therefore, to give an indication, a numerical order estimate is computed. We evaluate the results found after calculations on 


\begin{tabular}{|c|c|c|}
\hline & $E_{\mathrm{r}}(H)_{\text {band }}$ & $\frac{E_{\mathrm{r}}(H)_{\text {band }_{\mathrm{nL} / 2}}}{E_{\mathrm{r}}(H)_{\text {band }_{\mathrm{nL}}}}$ \\
\hline $\mathrm{nL}=72$ & $2.05 \cdot 10^{-3}$ & \\
\hline $\mathrm{nL}=144$ & $2.69 \cdot 10^{-4}$ & 7.6 \\
\hline $\mathrm{nL}=288$ & $3.65 \cdot 10^{-5}$ & 7.4 \\
\hline $\mathrm{nL}=576$ & $7.17 \cdot 10^{-6}$ & 5.1 \\
\hline
\end{tabular}

Table 4: Error measures on $H$ for different values of $\mathrm{nL}$ taken over a band between the latitudes $\phi=-60^{\circ}$ and $\phi=60^{\circ}$ on a global uniform lat-lon grid, where we prescribed the analytic solution outside the band.

a global reduced lat-lon grid. We ran four tests, each time doubling the value of $\mathrm{nL}(0)$ defined as the amount of grid cells in the longitudinal direction on the unreduced grid part. We begin with $\mathrm{nL}(0)=72$. The cell distribution in the unreduced grid part is uniform. We again coarsen our grid each time the cell width in the longitudinal direction projected onto the sphere is reduced with a factor two as compared to the preceding reduction. In case of our grids, this rule yields three or four reductions. To make sure that our grid is not too coarse in regions close to the poles, we also ran test on grids with $\mathrm{nL}(0)=288$ and $\mathrm{nL}(0)=576$ where three instead of four reductions were applied as was originally prescribed by the reduction rule. The error measures on $H, E_{\mathrm{r}}(H)$, are shown in Table 5. This time the max-norm is taken over the whole domain. The entries in the third column yield the order factor. Per grid we give the amount of reductions and their corresponding latitudes.

\begin{tabular}{|ll|c|c|}
\hline & & $E_{\mathrm{r}}(H)$ & $\frac{E_{\mathrm{r}}(H) \frac{\mathrm{nL}}{2}(0)}{E_{\mathrm{r}}(H)_{\mathrm{nL}(0)}}$ \\
\hline $\mathrm{nL}(0)=72, \quad 3$ reductions resp. at $\phi=60^{\circ}, 70^{\circ}, 80^{\circ}$ & $1.10 \cdot 10^{-2}$ & \\
\hline $\mathrm{nL}(0)=144, \quad 3$ reductions resp. at $\phi=60^{\circ}, 75^{\circ}, 82.5^{\circ}$ & $3.66 \cdot 10^{-3}$ & 3.0 \\
\hline $\mathrm{nL}(0)=288, \quad 4$ reductions resp. at $\phi=60^{\circ}, 75^{\circ}, 82.5^{\circ}, 86.25^{\circ}$ & $3.40 \cdot 10^{-3}$ & 1.1 \\
\hline $\mathrm{nL}(0)=576, \quad 4$ reductions resp. at $\phi=60^{\circ}, 75^{\circ}, 82.5^{\circ}, 86.25^{\circ}$ & $1.74 \cdot 10^{-3}$ & 2.0 \\
\hline $\mathrm{nL}(0)=288, \quad 3$ reductions resp. at $\phi=60^{\circ}, 75^{\circ}, 82.5^{\circ}$ & $1.77 \cdot 10^{-3}$ & 2.1 \\
\hline $\mathrm{nL}(0)=576, \quad 3$ reductions resp. at $\phi=60^{\circ}, 75^{\circ}, 82.5^{\circ}$ & $8.81 \cdot 10^{-4}$ & 2.0 \\
\hline
\end{tabular}

Table 5: Error measures on $H$ for different values of $\mathrm{nL}(0)$ taken over the whole domain on a global reduced lat-lon grid $(\mathrm{nP}=\mathrm{nL}(0) / 2)$, where grid coarsening is performed at the given latitudes.

First, the results show that the reduced grid approach leads to first order accuracy. This conclusion is valid as long as our grid is not too coarse in the polar areas. In case of $\mathrm{nL}(0)=288$ with four reductions, this condition is obviously not fulfilled resulting in almost no error reduction. As compared to unreduced grids, see for instance the entry $9.82 \cdot 10^{-3}$ from Table 2 and $1.09 \cdot 10^{-2}$ from Table 5, the reduced grid approach results in a small accuracy loss on coarse grids. The accuracy loss on finer grids will be larger since we find first order accuracy on a reduced latlon grid. However, its positive influence on the stability restriction for explitcit time stepping compromises its use. As long as we take special care as to guarantee an acceptable amount of grid cells next to the poles, the errors are sufficiently small for practical purposes. 
We here omit an order estimate for calculations on a combined grid. As we will later show, the results mimic the accuracy behavior found on the reduced lat-lon grids. Investigations related to the connection problem are reported in the next section.

4.1.2 Placement of the stereocap As nicely illustrated by Figure 9, in stereographic coordinates velocities over the poles behave normal and smoothly and hence can be approximated in much greater accuracy using a stereocap. However, we have also concluded that to cover the whole sphere a stereographic grid must be combined with for instance a lat-lon grid, creating a connection problem as examined in Section 3. Besides the question how this connection problem influences the accuracy, we wish to answer the question what value we should take for $\bar{\phi}$, which we defined in Section 3.1 as the latitudinal boundary of the uniform lat-lon region $R_{\mathrm{II}}$. We expect these questions to be related, since the larger $\bar{\phi}$ the smaller the cells in the connection band. We ran four tests on a combined unreduced grid, having $\mathrm{nL}=144$ points, i.e. with $\Delta \lambda=\Delta \phi=2.5^{\circ}$, where we gradually changed $\bar{\phi}$. Figure 11 shows the combined grids in case of the extreme values of $\bar{\phi}$. We coupled $x_{r}$ defined in Section 3.1 as the $x_{\mathrm{st}}$-coordinate of the top right-hand corner of the stereocap to $\bar{\phi}$, following $\phi_{x_{r}}=\bar{\phi}+\Delta \phi / 2$. $\phi_{x_{r}}$ denotes the latitude corresponding to the stereographic coordinates $\left(x_{\mathrm{st}}, y_{\mathrm{st}}\right)=\left(x_{r}, y_{r}\right)$. Table 6 displays the different error measures on $H$, $u$ and $U$ over five different regions, i.e. over the uniform lat-lon grid part, over the cells located at the equator, over the interface cells connecting the two grids, over the stereographic grid parts and over the cells next to the poles. Note that the interface cells, the cells located at the equator and the cells next to the poles are also included in the lat-lon grid part or the stereographic parts, see Section 3.1. $E_{\mathrm{r}}(H)$ again describes the max-norm of the relative error on $H . E_{\mathrm{a}}(u)$ and $E_{\mathrm{a}}(U)$ describe max-norms of the absolute error on $u$ and $U$, respectively.

\begin{tabular}{|c|c|c|c|c|c|}
\hline & $E_{\mathrm{r}}(H)_{\text {lat-lon grid part }}$ & $E_{\mathrm{r}}(H)_{\text {equator }}$ & $E_{\mathrm{r}}(H)_{\text {interface }}$ & $E_{\mathrm{r}}(H)_{\text {stereo grid part }}$ & $E_{\mathrm{r}}(H)_{\text {pole }}$ \\
\hline $\bar{\phi}=47.5^{\circ}$ & $1.40 \cdot 10^{-1}$ & $1.35 \cdot 10^{-1}$ & $4.56 \cdot 10^{-2}$ & $5.10 \cdot 10^{-2}$ & $3.86 \cdot 10^{-2}$ \\
\hline $\bar{\phi}=57.5^{\circ}$ & $7.58 \cdot 10^{-2}$ & $3.64 \cdot 10^{-2}$ & $3.27 \cdot 10^{-2}$ & $1.41 \cdot 10^{-2}$ & $7.14 \cdot 10^{-3}$ \\
\hline $\bar{\phi}=67.5^{\circ}$ & $6.53 \cdot 10^{-3}$ & $7.6 \cdot 10^{-5}$ & $6.53 \cdot 10^{-3}$ & $4.30 \cdot 10^{-3}$ & $1.43 \cdot 10^{-3}$ \\
\hline $\bar{\phi}=77.5^{\circ}$ & $2.48 \cdot 10^{-3}$ & $2.30 \cdot 10^{-3}$ & $2.31 \cdot 10^{-3}$ & $7.42 \cdot 10^{-4}$ & $3.00 \cdot 10^{-4}$ \\
\hline \multirow[t]{2}{*}{$\bar{\phi}=87.5^{\circ}$} & $1.29 \cdot 10^{-3}$ & $1.29 \cdot 10^{-3}$ & $6.67 \cdot 10^{-4}$ & $6.33 \cdot 10^{-4}$ & $6.00 \cdot 10^{-4}$ \\
\hline & $E_{\mathrm{a}}(u)_{\text {lat-lon grid part }}$ & $E_{\mathrm{a}}(u)$ equator & $E_{\mathrm{a}}(u)_{\text {interface }}$ & $E_{\mathrm{a}}(U)_{\text {stereo grid part }}$ & $E_{\mathrm{a}}(U)_{\text {pole }}$ \\
\hline $\bar{\phi}=47.5^{\circ}$ & 43.06 & 17.45 & 43.06 & 37.38 & $6.0 \cdot 10^{-2}$ \\
\hline $\bar{\phi}=57.5^{\circ}$ & 21.31 & 11.00 & 21.31 & 19.19 & $3.6 \cdot 10^{-2}$ \\
\hline $\bar{\phi}=67.5^{\circ}$ & 5.23 & 2.39 & 5.23 & 3.43 & $1.3 \cdot 10^{-2}$ \\
\hline $\bar{\phi}=77.5^{\circ}$ & 0.84 & 0.27 & 0.84 & 0.58 & $3.8 \cdot 10^{-4}$ \\
\hline $\bar{\phi}=87.5^{\circ}$ & 0.14 & 0.02 & 0.14 & 0.30 & $2.8 \cdot 10^{-4}$ \\
\hline
\end{tabular}

Table 6: Error measures on $H, u$, and $U$ for different values of $\bar{\phi}$ on four combined uniform latlon stereographic grids $(\mathrm{nL}=144)$. We give the errors $E_{\mathrm{r}}(H), E_{\mathrm{a}}(u)$ and $E_{\mathrm{a}}(U)$ over five different regions, i.e. over the uniform lat-lon grid part, over the cells located at the equator, over the interface cells connecting the two grids, over the stereographic grid parts and over the cells next to the poles.

As expected, Table 6 shows that it is best to make the stereocap as small as possible, restricting 
accuracy loss due to the connection problem at the grid interface. The influence of reducing the size of the interface cells is particularly visible when concentrating on the maximum absolute error of the velocities. We encounter an unavoidable accuracy reduction at the grid interface. However on grids with a small size stereocap this error is sufficiently small. Furthermore, both the errors on $H$ and $U$ are impressingly small at the poles. Comparing the overall error $E_{\mathrm{r}}(H)$ for $\bar{\phi}=77.5^{\circ}$ with the second entry from Table 5 , we see that our calculations on a combined grid with a stereocap result in the same overall accuracy as the calculations on a compatible reduced grid. Note that this conclusion is true for modest and small sized stereocaps. For large stereocaps the interface cells become too distorted.
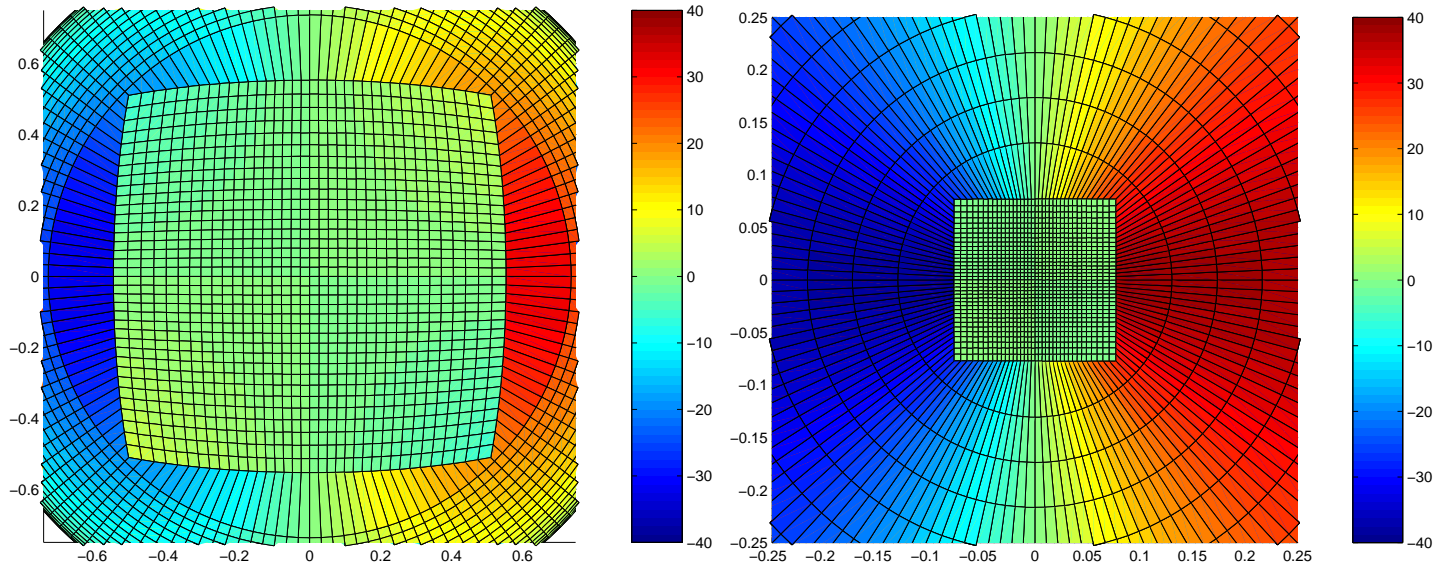

Figure 11: Projection of two combined grids $(\mathrm{nL}=144)$ onto the cartesian $(\mathrm{x}, \mathrm{y})$-plane $(\mathrm{z}=0)$, where the stereocap in the right picture is moved closer to the pole. $\bar{\phi}=47.5^{\circ}$ (left picture) and $\bar{\phi}=87.5^{\circ}$ (right picture). Along the axes, the $x$ - and $y$-coordinate are given as multiples of the earth radius.

4.1.3 A combined grid with realistic refinement Figure 11 shows that our conclusion should be handled with a little consideration. When performance issues are important, the resolution increase on the stereocap due to size reduction can lead to a cut-back on the time-step caused by stability restrictions. However, this problem is easily resolved when we add the reduced grid approach to our combined grid. To show this, we end our numerical section on test case two of [26] by giving the results of a test on a combined reduced grid with realistic refinements. The stereocap is placed such that $\bar{\phi}=85.625^{\circ}, \mathrm{nL}(0)=576$ and $\mathrm{nP}=288$. We apply 3 reductions, one at $60^{\circ}$, one at $75^{\circ}$ and one at $82.5^{\circ}$.

The results confirm our expectations. We find a maximum relative error on $H$ over our whole domain of $E_{\mathrm{r}}(H)=8.6 \cdot 10^{-4}$ and a maximum absolute error on $u, U$ of $E_{\mathrm{a}}(u, U)=0.092$. These errors show that a combined grid provides a good alternative to a global reduced lat-lon grid, see Table 5 case $\mathrm{nL}(0)=576$ with three reductions. This conclusion holds in particular, when the CFL-restriction demands a too coarse lat-lon grid around the poles to maintain an acceptable time-step. This follows when comparing the smallest grid sizes found on the two different grid types. Note that in either case the smallest stepsize is found next to the poles. For the combined grid, the smallest grid size on the globe approximately reads

$$
\frac{\sqrt{2} \pi a \cos \bar{\phi}}{\mathrm{nL}_{\text {interface }}} \text {. }
$$

On a reduced lat-lon grid, the smallest grid size reads

$$
\frac{2 \pi a \cos \left(90^{\circ}-\Delta \phi\right)}{\mathrm{nL}_{\text {interface }}} \text {. }
$$


Based on (4.6) and (4.7), we give the smallest grid size ratio for $\Delta \phi=0.625^{\circ}, \mathrm{nL}_{\text {interface }}=72$ and $\bar{\phi}=85.625^{\circ}$. The ratio reads

$$
\frac{\frac{1}{2} \sqrt{2} \cos \bar{\phi}}{\cos \left(90^{\circ}-\Delta \phi\right)} \approx 4.95 .
$$

For explicit integration methods this ratio suggests a difference in computing time of approximately a factor five in favor of the combined grid. Note that the time step restriction can indeed be encountered in practical situations, since high velocity components do occur in the polar areas.

\section{Concluding Remarks}

Spectral methods currently dominate the field of approximation methods used in global circulation modelling. Since spectral methods become relatively expensive on fine grids, the demand for higher grid resolution and the better prospects for parallelization and local grid refinement has renewed interest in gridpoint methods. In this paper we have studied a sophisticated finite-volume scheme for the spatial discretization of the SWEs in spherical geometry, viz. Osher's scheme [16] using the P-variant of Hemker and Spekreijse [10] for the integration path in the flux evaluation and third order upwinding for the determination of the constant states. The scheme's higher order accuracy, its robustness and its apprehension for the characteristic directions associated with the nonlinear equations, makes it a natural competitor to spectral methods for computations on fine grids.

We have paid special attention to the pole singularity and the associated CFL-restriction. We have examined a combined grid to thoroughly alleviate the associated problems. This combined grid connects a stereographic grid in the polar regions with a lat-lon grid used at low latitudes. We have found that it is best to keep the size of the stereocap rather small so as to minimize connection errors at the grid interface. Since a small stereocap involves small grid sizes at and near the cap, grid reduction in the lat-lon part can be used when it is needed to avoid very small grid sizes. In this manner the time step limitation for explicit integration methods emanating from the pole problem can be significantly reduced. Therefore, the resulting combined grid is advocated to be used together with an explicit integration scheme. In case time step stability plays a minor role, or when an implicit type integration method is used, we advocate to use only a lat-lon grid, possibly reduced, because this approach is simpler. However, on lat-lon grids the singularity remains so that in case of flow over the poles the grid should be fine enough to resolve it.

Our findings are based on test cases one, two and six of the standard test set from [26]. To save space we have shown results for test case two only. In the near future we will present results on time integration aspects using the spatial discretizations described in the current paper.

\section{ACKNOWLEDGEMENT}

We gratefully acknowledge David Williamson for his clarifying discussions on the pole problem. The investigations were in part supported by the Research Councel for Earth and Lifesciences (ALW) with financial aid from the Netherlands Organization for Scientific Research (NWO).

\section{REFERENCES}

1. J.G. Blom, W. Hundsdorfer, and J.G. Verwer. Vectorization aspects of a spherical advection scheme on a reduced grid. Technical Report NM-R9418, CWI, Amsterdam, 1994.

2. G.L. Browning, J.J. Hack, and P.N. Swarztrauber. A comparison of three numerical methods for solving differential equations on the sphere. Mon. Wea. Rev., 117:1058-1075, 1989.

3. K. Cassirer, R. Hess, C. Jablonowski, and W. Joppich. The shallow water test cases for a global model with documentation of the results. Arbeitspapiere der GMD 999, GMD, 1996.

4. T. Davies and J.C.R. Hunt. New developments in numerical weather prediction. In K.W. Morton and M.J. Baines, editors, Numerical methods for fluid dynamics V. Clarendon Press, Oxford, 1995.

5. ECMWF. ECMWF forecast model documentation. European Centre for Medium-Range Weather Forecasts, Shinefield Park, England, ECMWF Research Manual edition, 1988. 
6. E.B. Eliasen, B. Machenhauer, and E. Rasmussen. On a numerical method for integration of the hydrodynamical equations with a spectral representation of the horizontal fields. Report No. 2, Institut for Teoretisk Meteorologi, University of Copenhagen, 1970.

7. B. Engquist and S. Osher. Stable and entropy satisfying approximations for transonic flow calculations. Math. Comp., 34:45-75, 1980.

8. B. Engquist and S. Osher. One sided difference approximations for nonlinear conservation laws. Math. Comp., 36:321-352, 1981.

9. G.J. Haltiner and R.T. Williams. Numerical prediction and dynamic meteorology. Wiley, 2nd edition, 1980.

10. P.W. Hemker and S.P. Spekreijse. Multiple grid and Osher's scheme for the efficient solution of the steady state Euler equations. Appl. Numer. Math., 2:475-493, 1986.

11. C. Hirsch. Numerical computation of internal and external flows, volume 2: Computational methods for inviscid and viscous flow. John Wiley \& Sons, Chichester, 1990.

12. J.R. Holton. An introduction to dynamic meteorology. Academic Press, 1992.

13. J.L. Lions, R. Temam, and S. Wang. New formulations of the primitive equations of atmosphere and applications. Nonlinearity, 5:237-288, 1992.

14. S.A. Orszag. Transform method for calculation of vector coupled sums: Application to the spectral form of the vorticity equation. J. Atmos. Sci., 27:890-895, 1970.

15. S. Osher and S. Chakravarthy. Upwind schemes and boundary conditions with applications to Euler equations in general geometries. J. Comp. Phys., 50:447-481, 1983.

16. S. Osher and F. Solomon. Upwind difference schemes for hyperbolic systems of conservation laws. Math. Comp., 38:339-374, 1982.

17. N.A. Phillips. A map projection system suitable for large-scale numerical weather prediction. J. Meteor. Soc. Japan, 75:262-267, 1957.

18. S.P. Spekreijse. Multigrid solution of the steady Euler equations. Number 46 in CWI Tracts. CWI, Amsterdam, 1988

19. B. Spotz, M. Taylor, and P. Swarztrauber. Shallow water equations on the sphere. http://www.scd.ucar.edu/css/staff/spotz/research/swell.html\#STM.

20. G. Starius. Composite mesh difference methods for elliptic and boundary value problems. Numer. Math., 28:243-258, 1977.

21. J.J. Stoker and E. Isaacson. Final report 1. Technical Report IMM 407, Courant Institute of Mathematical Sciences, New York University, 1975.

22. B. van Leer. Towards the ultimate conservative difference scheme. III. Upstream-centered finite-difference schemes for ideal compressible flow. J. Comput. Phys., 23:276-299, 1977.

23. D.L. Williamson. Numerical Methods Used in Atmospheric Models, volume II of GARP Publication Series No. 17, chapter Difference Approximations for fluid flow on a sphere, pages 51-120. World Meteorological Organization, Geneva, 1979.

24. D.L. Williamson. Review of numerical approaches for modeling global transport. In H. van Dop and G. Kallos, editors, Air Pollution Modeling and its application IX, Nato Challenges of Modern Society, pages 377-394, New York, 1992. Plenum Press.

25. D.L. Williamson and G.L. Browning. Comparison of grids and difference approximations for numerical weather prediction over a sphere. J. Appl. Meteor., 12:264-274, 1973.

26. D.L. Williamson, J.B. Drake, J.J. Hack, R. Jacob, and P.N. Swarztrauber. A standard test set for numerical approximations to the shallow water equations in spherical geometry. J. Comp. Phys., 102:211-224, 1992. 


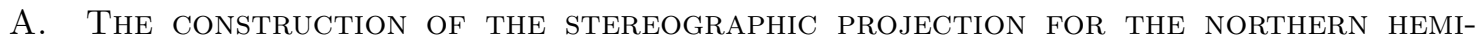
SPHERE

In this appendix we construct the relations between the stereographic $\left(x_{\mathrm{st}}, y_{\mathrm{st}}\right)$ and spherical coordinates $(\lambda, \phi)$. Consider a point $\underline{r}=(\lambda, \phi, a)$ at the sphere on the northern hemisphere. Further consider the half-plane $S_{\lambda}$ defined as the plane with $\lambda$ constant and consider the stereographic plane defined as the plane located at and locally tangent to the sphere at the pole. Project then from the south pole point $\underline{r}=(\lambda, \phi, a)$ onto the intersection of the plane $S_{\lambda}$ and the stereographic plane, see figure 12. We denote this projection point as $\underline{r}_{\mathrm{st}}=\left(x_{\mathrm{st}}, y_{\mathrm{st}}\right)$, see Figure 12 .

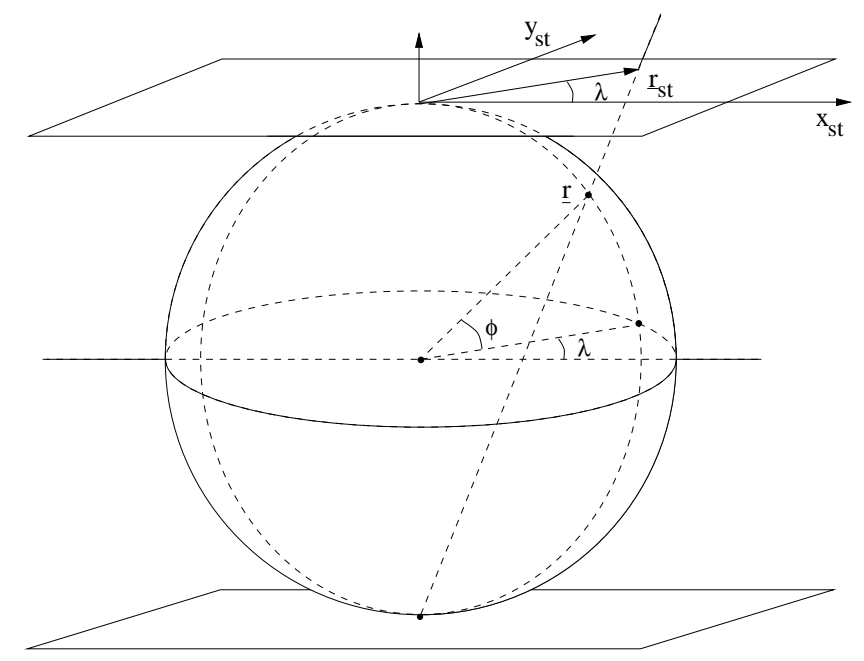

Figure 12: The projection of the northern hemisphere onto the stereographic plane in the polar case.

From figure 13(a) it can then be derived that $\left|r_{\text {st }}\right|=a m \cos \phi$ with $m=2 /(1+\sin \phi)$. Further let the positive stereographic $x_{\mathrm{st}}$-axis correspond with the intersection of the stereographic plane

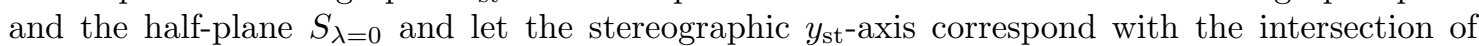
the stereographic plane and the half-plane $S_{\lambda=\pi / 2}$. From figure 13(b) then easily follows that $\underline{r}_{\mathrm{st}}=\left(\left|r_{\mathrm{st}}\right| \cos \lambda,\left|r_{\mathrm{st}}\right| \sin \lambda\right)$.

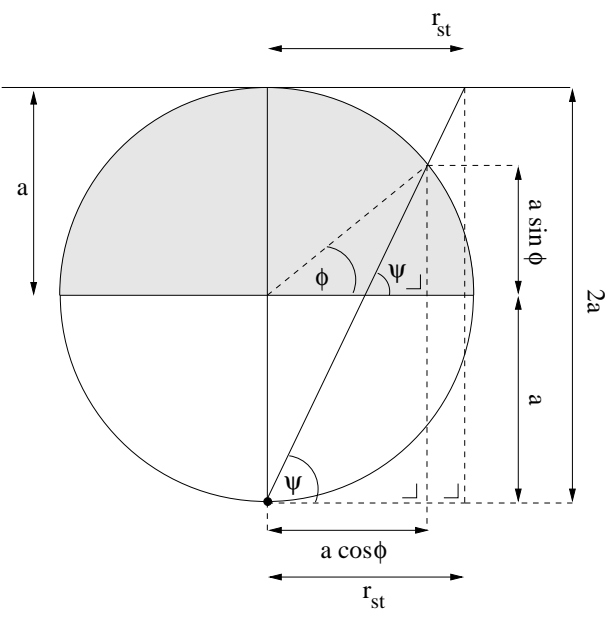

(a) Cross-section of the sphere with radius a and the surfaces $S_{\lambda}$ and $S_{\lambda+\pi}$

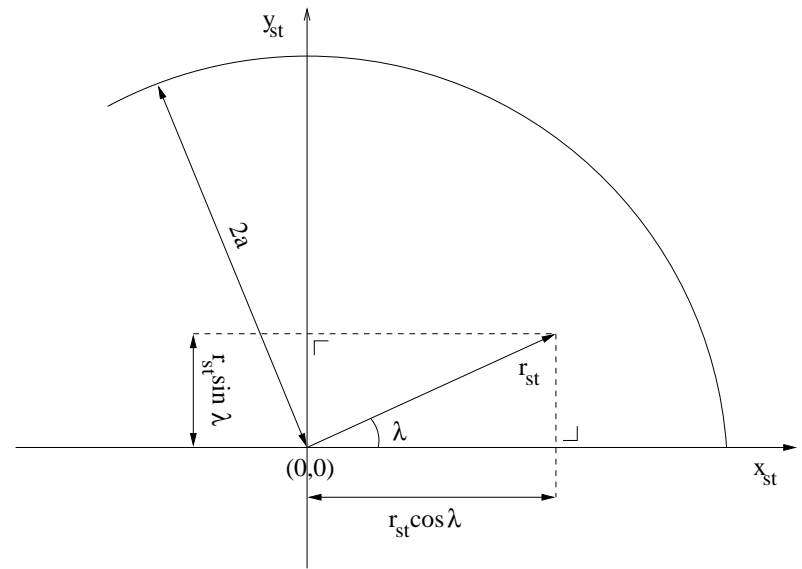

(b) The stereographic plane for the northern hemisphere projection in the polar case.

Figure 13: The geometry of the stereographic mapping (northern hemisphere projection). 
The relations between the stereographic and spherical coordinates then yield

$$
\begin{aligned}
& x_{\mathrm{st}}=a m \cos \phi \cos \lambda, \quad-\pi / 2<\phi<\pi / 2,0 \leq \lambda<2 \pi, \\
& y_{\mathrm{st}}=a m \cos \phi \sin \lambda, \quad-\pi / 2<\phi<\pi / 2,0 \leq \lambda<2 \pi,
\end{aligned}
$$

where $\mathrm{m}$ is the map factor

$$
m=\frac{2}{1+\alpha \sin \phi},
$$

with $\alpha$ distinguishing between the northern $(\alpha=1)$ and the southern hemisphere projection $(\alpha=$ $-1)$. A clear description of the stereographic projection can be found in [23].

\section{B. Construction of the stereographic formulation of the SWEs from the SPheri-} CAL FORMULATION OF THE SWES

In this appendix we construct the SWEs in stereographic coordinates from the SWEs in spherical coordinates. Note that this construction is valid on the whole sphere with exception of the poles. To derive the stereographic formulation of the SWEs on the whole sphere a thorough tensor analysis is necessary. We start with the introduction of a few useful relations between both coordinate systems. Between the spherical $(\lambda, \phi)$ and the stereographic coordinates $\left(x_{\mathrm{st}}, y_{\mathrm{st}}\right)$ we have

$$
\begin{aligned}
& x_{\mathrm{st}}=a m \cos \phi \cos \lambda, \\
& y_{\mathrm{st}}=a m \cos \phi \sin \lambda,
\end{aligned}
$$

with

$$
m=\frac{2}{1+\alpha \sin \phi} .
$$

For the velocity fields $\underline{u}=(u, v)$ and $\underline{U}=(U, V)$ we have

$$
\begin{aligned}
U & =-u \sin \lambda-\alpha v \cos \lambda, \\
V & =u \cos \lambda-\alpha v \sin \lambda .
\end{aligned}
$$

For the relations (B.1)-(B.5) we can derive their inverses

$$
\begin{aligned}
\phi & =\alpha \arcsin \left[\frac{4 a^{2}-x_{\mathrm{st}}^{2}-y_{\mathrm{st}}^{2}}{4 a^{2}+x_{\mathrm{st}}^{2}+y_{\mathrm{st}}^{2}}\right], \\
\lambda & =\arctan \left(\frac{y_{\mathrm{st}}}{x_{\mathrm{st}}}\right),
\end{aligned}
$$

and

$$
\begin{aligned}
& u=-U \sin \lambda+V \cos \lambda, \\
& v=-\alpha U \cos \lambda-\alpha V \sin \lambda .
\end{aligned}
$$

Remember that the spherical velocity field components $(u, v)$ are defined as

$$
\begin{aligned}
& u=a \cos \phi \dot{\lambda}, \\
& v=a \dot{\phi},
\end{aligned}
$$

where $\dot{\lambda}, \dot{\phi}$ denote the substantial or total time derivatives $\frac{\mathrm{d} \lambda}{\mathrm{d} t}, \frac{\mathrm{d} \phi}{\mathrm{d} t}$. For the stereographic velocity field components $(U, V)$ defined as $\left(m^{-1} \dot{x}_{\mathrm{st}}, m^{-1} \dot{y}_{\mathrm{st}}\right)$, hold

$$
\begin{aligned}
U & =-a \dot{\lambda} \cos \phi \sin \lambda-\alpha a \dot{\phi} \cos \lambda, \\
V & =a \dot{\lambda} \cos \phi \cos \lambda-\alpha a \dot{\phi} \sin \lambda .
\end{aligned}
$$


Finally, we also need the inverses of these relations,

$$
\begin{aligned}
\dot{\lambda} & =\frac{1}{a \cos \phi}(-U \sin \lambda+V \cos \lambda), \\
\dot{\phi} & =\frac{\alpha}{a}(-U \cos \lambda-V \sin \lambda) .
\end{aligned}
$$

With the relations (B.1)-(B.10) we are able to derive the SWEs in stereographic coordinates from the SWEs in spherical coordinates. In this last coordinate system the SWEs in advective form are given by

$$
\begin{aligned}
\frac{\mathrm{d} H}{\mathrm{~d} t}+H \nabla \cdot \underline{u} & =0, \\
\frac{\mathrm{d} u}{\mathrm{~d} t}-\left(f+\frac{u}{a} \tan \phi\right) v+\frac{g}{a \cos \phi} \frac{\partial h}{\partial \lambda} & =0, \\
\frac{\mathrm{d} v}{\mathrm{~d} t}+\left(f+\frac{u}{a} \tan \phi\right) u+\frac{g}{a} \frac{\partial h}{\partial \phi} & =0,
\end{aligned}
$$

where

$$
\nabla \cdot \underline{u}=\frac{1}{a \cos \phi}\left[\frac{\partial u}{\partial \lambda}+\frac{\partial(v \cos \phi)}{\partial \phi}\right]
$$

and

$$
\frac{\mathrm{d} H}{\mathrm{~d} t}=\frac{\partial H}{\partial t}+\underline{u} \cdot \nabla H
$$

with

$$
\nabla H=\left(\frac{1}{a \cos \phi} \frac{\partial H}{\partial \lambda}, \frac{1}{a} \frac{\partial H}{\partial \phi}\right)
$$

We are interested in the stereographic formulation of the SWEs in flux-form. The derivation steps are described below. We start with the equation of motion in $x_{\mathrm{st}}$-direction

$$
\begin{aligned}
& \dot{U} \stackrel{\text { step }}{=}{ }^{1} \quad\left(\left(f+\frac{u}{a} \tan \phi\right) v-\frac{g}{a \cos \phi} \frac{\partial h}{\partial \lambda}\right) \cdot-\sin \lambda+\left(\left(f+\frac{u}{a} \tan \phi\right) u+\frac{g}{a} \frac{\partial h}{\partial \phi}\right) \cdot \alpha \cos \lambda-V \dot{\lambda} \\
& \text { step }^{2} \alpha f V+(\alpha \sin \phi-1) \dot{\lambda} V-m g \frac{\partial h}{\partial x_{\mathrm{st}}} \\
& \stackrel{\text { step }}{{ }^{3}} \alpha f V-\frac{\left(\alpha x_{\mathrm{st}} V-y_{\mathrm{st}} U\right) V}{2 a^{2}}-m g \frac{\partial h}{\partial x_{\mathrm{st}}} \text {. }
\end{aligned}
$$

Step 1 Through (B.4), (B.5), (B.7) and (B.8) we can derive that

$$
\begin{aligned}
\dot{U} & =-\dot{u} \sin \lambda-\alpha \dot{v} \cos \lambda-u \cos \lambda \dot{\lambda}+\alpha v \sin \lambda \dot{\lambda} \\
& =-\dot{u} \sin \lambda-\alpha \dot{v} \cos \lambda-V \dot{\lambda}, \\
\dot{V} & =\dot{u} \cos \lambda-\alpha \dot{v} \sin \lambda-u \sin \lambda \dot{\lambda}-\alpha v \cos \lambda \dot{\lambda} \\
& =\dot{u} \cos \lambda-\alpha \dot{v} \sin \lambda+U \dot{\lambda} .
\end{aligned}
$$

Step 2 This step consists of two substeps.

Step $2 a$ Using (B.5) and (B.9), we first rewrite $\left(f+\frac{u}{a} \tan \phi\right) v \cdot-\sin \lambda+\left(f+\frac{u}{a} \tan \phi\right) u \cdot \alpha \cos \lambda$ in terms of the stereographic coordinates

$$
\begin{aligned}
\left(f+\frac{u}{a} \tan \phi\right) v \cdot-\sin \lambda+\left(f+\frac{u}{a} \tan \phi\right) u \cdot \alpha \cos \lambda & =\left(f+\frac{u}{a} \tan \phi\right)(-v \sin \lambda+\alpha u \cos \lambda) \\
& =\alpha f V+\alpha \sin \phi \dot{\lambda} V .
\end{aligned}
$$


Step $2 b$ Then, through (B.1)-(B.3), we rewrite $\frac{g}{a \cos \phi} \sin \lambda \frac{\partial h}{\partial \lambda}+\frac{\alpha g}{a} \cos \lambda \frac{\partial h}{\partial \phi}$ in terms of the stereographic coordinates

$$
\frac{g \sin \lambda}{a \cos \phi} \frac{\partial h}{\partial \lambda}+\frac{\alpha g \cos \lambda}{a} \frac{\partial h}{\partial \phi}=\frac{g}{a}\left(\frac{\sin \lambda}{\cos \phi}\left(\frac{\partial h}{\partial x_{\mathrm{st}}} \frac{\partial x_{\mathrm{st}}}{\partial \lambda}+\frac{\partial h}{\partial y_{\mathrm{st}}} \frac{\partial y_{\mathrm{st}}}{\partial \lambda}\right)+\alpha \cos \lambda\left(\frac{\partial h}{\partial x_{\mathrm{st}}} \frac{\partial x_{\mathrm{st}}}{\partial \phi}+\frac{\partial h}{\partial y_{\mathrm{st}}} \frac{\partial y_{\mathrm{st}}}{\partial \phi}\right)\right)
$$

with

$$
\left(\begin{array}{ll}
\frac{\partial x_{\mathrm{st}}}{\partial \lambda} & \frac{\partial x_{\mathrm{st}}}{\partial \phi} \\
\frac{\partial y_{\mathrm{st}}}{\partial \lambda} & \frac{\partial y_{\mathrm{st}}}{\partial \phi}
\end{array}\right)=\left(\begin{array}{cc}
-a m \cos \phi \sin \lambda & a \frac{\mathrm{d} m}{\mathrm{~d} \phi} \cos \phi \cos \lambda-a m \sin \phi \cos \lambda \\
a m \cos \phi \cos \lambda & a \frac{\mathrm{d} m}{\mathrm{~d} \phi} \cos \phi \sin \lambda-a m \sin \phi \sin \lambda
\end{array}\right)
$$

and

$$
\frac{\mathrm{d} m}{\mathrm{~d} \phi}=-\frac{2 \alpha \cos \phi}{(1+\alpha \sin \phi)^{2}}=-\frac{m \alpha \cos \phi}{(1+\alpha \sin \phi)}
$$

When we combine (B.11)-(B.13) we find

$$
\begin{aligned}
& \frac{g}{a \cos \phi} \sin \lambda \frac{\partial h}{\partial \lambda}+\frac{\alpha g}{a} \cos \lambda \frac{\partial h}{\partial \phi}= \\
& =g\left(-m \sin ^{2} \lambda-\alpha m \sin \phi \cos ^{2} \lambda+\frac{-m \cos ^{2} \phi \cos ^{2} \lambda}{(1+\alpha \sin \phi)}\right) \frac{\partial h}{\partial x_{\mathrm{st}}}+ \\
& \quad+g\left(m \cos \lambda \sin \lambda-\alpha m \sin \phi \cos \lambda \sin \lambda-\frac{m \cos ^{2} \phi}{1+\alpha \sin \phi} \sin \lambda \cos \lambda\right) \frac{\partial h}{\partial y_{\mathrm{st}}} \\
& =-m g \frac{\partial h}{\partial x_{\mathrm{st}}} .
\end{aligned}
$$

Step 3 We focus on the total derivative $\dot{\lambda}$. Multiply $\dot{\lambda}$ with $\alpha \sin \phi-1$. Through (B.1), (B.2) and (B.10) then follows

$$
\begin{aligned}
(\alpha \sin \phi-1) \dot{\lambda} & =\left(-\frac{(1-\alpha \sin \phi)}{a^{2} m \cos ^{2} \phi}\left(x_{\mathrm{st}} V-y_{\mathrm{st}} U\right)\right) \\
& =-\frac{(1-\alpha \sin \phi)(1+\alpha \sin \phi)}{2 a^{2} \cos ^{2} \phi}\left(x_{\mathrm{st}} V-y_{\mathrm{st}} U\right) \\
& =-\frac{1}{2 a^{2}}\left(x_{\mathrm{st}} V-y_{\mathrm{st}} U\right) .
\end{aligned}
$$

In a similar way we can derive the equation of motion in the $y_{\text {st }}$-direction. This equation reads

$$
\dot{V}=-\alpha f U+\frac{1}{2 a^{2}}\left(x_{\mathrm{st}} V-y_{\mathrm{st}} U\right)-m g \frac{\partial h}{\partial y_{\mathrm{st}}} .
$$

The continuity equation remains. In terms of the stereographic coordinates this equation is described as

$$
\dot{H}+H \nabla \cdot \underline{U}=0
$$

with

$$
\nabla \cdot \underline{U} \stackrel{\text { step }}{=} m^{2}\left[\frac{\partial}{\partial x_{\mathrm{st}}}\left(\frac{U}{m}\right)+\frac{\partial}{\partial y_{\mathrm{st}}}\left(\frac{V}{m}\right)\right],
$$

where Step 4 is applied. 
Step 4 For the divergence operator holds, by definition,

$$
\left(\nabla_{\text {sphere }} \cdot \underline{u}\right)=\left(\nabla_{\text {st }} \cdot \underline{U}\right),
$$

where $\left(\nabla_{\text {sphere }} \cdot \underline{u}\right)$ is defined as the divergence operator in spherical coordinates

$$
\nabla_{\text {sphere }} \cdot \underline{u} \equiv \frac{1}{a \cos \phi}\left[\frac{\partial u}{\partial \lambda}+\frac{\partial v \cos \phi}{\partial \phi}\right] .
$$

We need to derive the divergence operator in terms of the stereographic coordinates. We have

$$
\nabla_{\text {sphere }} \cdot(A \underline{u}) \equiv \frac{1}{a \cos \phi}\left[\frac{\partial}{\partial \lambda}(A u)+\frac{\partial}{\partial \phi}(A v \cos \phi)\right] .
$$

In combination with equations (B.7), (B.8), (B.12) and (B.13), we find

$$
\nabla_{\text {sphere }} \cdot(A \underline{u})=m \frac{\partial}{\partial x_{\mathrm{st}}}(A U)+m \frac{\partial}{\partial y_{\mathrm{st}}}(A V)+\frac{\alpha \sin \phi-1}{a \cos \phi}(\cos \lambda A U+\sin \lambda A V) .
$$

To further explore the last term in this equation, we have to realize that

$$
\begin{aligned}
\frac{\partial \phi}{\partial x_{\mathrm{st}}} & =-\frac{\alpha \cos \lambda}{a m}, \\
\frac{\partial \phi}{\partial y_{\mathrm{st}}} & =-\frac{\alpha \sin \lambda}{a m},
\end{aligned}
$$

where we applied equation (B.6). Together with (B.13) we then find

$$
\begin{aligned}
\frac{\partial m}{\partial x_{\mathrm{st}}} & =\frac{\cos \lambda(1-\alpha \sin \phi)}{a \cos \phi}, \\
\frac{\partial m}{\partial y_{\mathrm{st}}} & =\frac{\sin \lambda(1-\alpha \sin \phi)}{a \cos \phi} .
\end{aligned}
$$

So, the last term in equation (B.15) yields

$$
-\frac{(1-\alpha \sin \phi)}{a \cos \phi}(\cos \lambda A U+\sin \lambda A V)=-\left(A U \frac{\partial m}{\partial x_{\mathrm{st}}}+A V \frac{\partial m}{\partial y_{\mathrm{st}}}\right) .
$$

When we combine this equation with the equations (B.14) and (B.15), we find for the divergence operator in stereographic coordinates

$$
\nabla_{\mathrm{st}} \cdot(A \underline{U}) \equiv m^{2} \frac{\partial}{\partial x_{\mathrm{st}}}\left(\frac{A U}{m}\right)+m^{2} \frac{\partial}{\partial y_{\mathrm{st}}}\left(\frac{A V}{m}\right)
$$

We summarize the advective form of the SWEs in stereographic coordinates

$$
\begin{aligned}
\dot{H} & =-H \nabla \cdot \underline{U}, \\
\dot{U} & =\alpha f V-\frac{\left(x_{\mathrm{st}} V-y_{\mathrm{st}} U\right) V}{2 a^{2}}-m g \frac{\partial h}{\partial x_{\mathrm{st}}}, \\
\dot{V} & =-\alpha f U+\frac{1}{2 a^{2}}\left(x_{\mathrm{st}} V-y_{\mathrm{st}} U\right)-m g \frac{\partial h}{\partial y_{\mathrm{st}}},
\end{aligned}
$$

where, by definition, the total derivative reads

$$
\begin{aligned}
\dot{H} & =\frac{\partial H}{\partial t}+\dot{x_{\mathrm{st}}} \frac{\partial H}{\partial x}+\dot{y_{\mathrm{st}}} \frac{\partial H}{\partial y_{\mathrm{st}}} \\
& =\frac{\partial H}{\partial t}+m U \frac{\partial H}{\partial x_{\mathrm{st}}}+m V \frac{\partial H}{\partial y_{\mathrm{st}}}
\end{aligned}
$$


and for the divergence holds

$$
\nabla \cdot \underline{U} \equiv m^{2}\left[\frac{\partial}{\partial x_{\mathrm{st}}}\left(\frac{U}{m}\right)+\frac{\partial}{\partial y_{\mathrm{st}}}\left(\frac{V}{m}\right)\right] .
$$

Finally, we combine the equations (B.16)-(B.20) to find the SWEs in flux-form,

$$
\begin{aligned}
\frac{\partial H}{\partial t}+\nabla \cdot(H \underline{U}) & =0 \\
\frac{\partial H U}{\partial t}+\nabla \cdot(H U \underline{U}) & =\left[\alpha f-\frac{\left(x_{\mathrm{st}} V-y_{\mathrm{st}} U\right)}{2 a^{2}}\right] H V-m g H \frac{\partial h}{\partial x_{\mathrm{st}}}, \\
\frac{\partial H V}{\partial t}+\nabla \cdot(H V \underline{U}) & =-\left[\alpha f-\frac{\left(x_{\mathrm{st}} V-y_{\mathrm{st}} U\right)}{2 a^{2}}\right] H U-m g H \frac{\partial h}{\partial y_{\mathrm{st}}} .
\end{aligned}
$$

Remember that our derivation is valid on the whole sphere with exception of the poles.

\section{Construction of the Osher flux}

In this appendix we describe the construction of the Osher flux. We start with the description of this flux for a general hyperbolic system of equations in $\mathbb{R}^{3}$ and then zoom in on the system of shallow water equations. The first part of this appendix is based on the article of Osher and Solomon [16].

Consider a general hyperbolic system of conservation laws in one dimension

$$
\frac{\partial \underline{q}}{\partial t}+\frac{\partial \underline{f}(\underline{q})}{\partial x}=0
$$

where $\underline{q}$ defines the state variable $\underline{q}=\left(q_{1}, q_{2}, q_{3}\right)^{T} \in \mathbb{R}^{3}$ and $\underline{f}$ defines the flux in $x$-direction. The system (C.1) is called hyperbolic, when the eigenvalues $\lambda_{k}$ of the Jacobian matrix $A$ of the flux $\underline{f}$ with respect to $\underline{q}, A=\partial \underline{f} / \partial \underline{q}$, are real and the corresponding eigenvectors $\underline{r}_{k}$ span the state space $\mathbb{R}^{3}$. Note that the Jacobian matrix $A$ depends on the state variable $\underline{q}$.

In a finite volume discretization of system (C.1), an approximation of the flux $\underline{f}(\underline{q})$ across each cell boundary is needed.

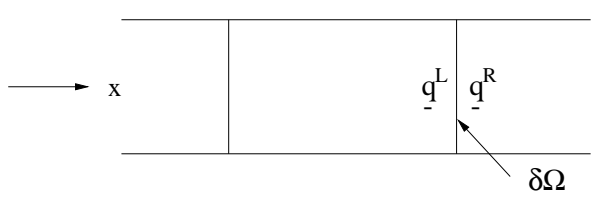

Figure 14: Situation at a cell boundary $\delta \Omega$.

Let $\delta \Omega$ be such a cell boundary ( $x=$ constant) and assume that respectively at the left and right of this boundary, constant states $q^{\mathrm{L}}$ and $q^{\mathrm{R}}$ are defined, see Figure 14 . We then approximate the resulting flux $\underline{f}$ across this boundary with Osher's flux, which is given as

$$
\underline{F}_{(\mathrm{O})}\left(\underline{q}^{\mathrm{L}}, \underline{q}^{\mathrm{R}}\right)=\frac{1}{2}\left(\underline{f}\left(\underline{q}^{\mathrm{L}}\right)+\underline{f}\left(\underline{q}^{\mathrm{R}}\right)\right)-\frac{1}{2} \int_{\underline{q}^{\mathrm{L}}}^{\underline{q}^{\mathrm{R}}}|A(\underline{q})| d \underline{q} .
$$

The absolute value of the Jacobian matrix $A$ is here defined by $|A|=P|\Lambda| P^{-1}$, where $P$ and $\Lambda$ result from diagonalizing the Jacobian matrix as $A=P \Lambda P^{-1}$. Note that, because of the system's hyperbolic character, the matrices $P$ and $P^{-1}$ exist.

The structure of the Osher flux (C.2) originates from a generalization of the Engquist and Osher flux $[7,8]$, which was developed for non-linear scalar conservation laws. In contrast to this flux, the Osher flux (C.2) is not uniquely determined. The path of integration between $\underline{q}^{\mathrm{L}}$ and $\underline{q}^{\mathrm{R}}$ in the state space $\mathbb{R}^{3}$ can be chosen in different ways, significantly influencing the properties of the resulting scheme. Osher made a natural choice for his path of integration, leading to his famous both elegant and well-applicable flux. 
Osher's path $\Gamma$ is composed of subcurves $\Gamma_{k}$ which are based on the eigenvectors $\underline{r}_{k}$ of the Jacobian matrix $A$, i.e.

$$
\Gamma=\bigcup_{k=1}^{3} \Gamma_{k}
$$

where $\Gamma_{k}$ is parameterized as

$$
\Gamma_{k}=\left\{\underline{q}^{k}(s): \frac{d \underline{q}^{k}}{d s}=\underline{r}_{k} \text { with } 0 \leq s \leq s_{k}\right\}
$$

and $\underline{q}_{\mathrm{b}}^{k}(0)$ and $\underline{q}_{\mathrm{e}}^{k}\left(s_{k}\right)$ denote respectively the begin and end point of this subcurve. Subcurves defined in this way correspond to rarefaction or compression wave solutions of system (C.1). The subcurves $\Gamma_{k}$ are passed in order of increasing corresponding eigenvalues $\lambda_{k}$, following the $\mathrm{P}$ (hysical)-variant proposed by Hemker and Spekreijse [10] to improve efficiency. Originally, Osher proposed to move along the subcurves $\Gamma_{k}$ in order of decreasing corresponding eigenvalues $\lambda_{k}$ (O(sher)-variant). Using hyperbolicity and the implicit function theorem, it can be shown that exactly one Osher path exists [16] for both the $\mathrm{P}$ - and O-variant. A schematic representation of the P-variant Osher path is given in Figure 15. The states $\underline{q}^{1 / 3}$ and $\underline{q}^{2 / 3}$ denote the unique intersection points between the different subcurves $\Gamma_{k}$. At the end of this section their exact value is given.

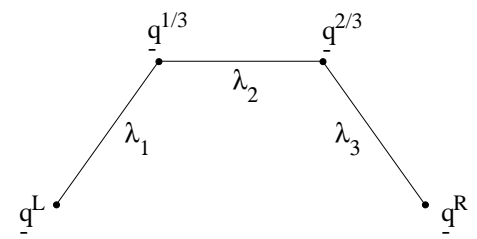

Figure 15: A schematic representation of the Osher path $\Gamma$ in case of the P-variant $\left(\lambda_{1} \leq \lambda_{2} \leq \lambda_{3}\right)$.

Along a subcurve $\Gamma_{k}$ the evaluation of the integral in (C.2) turns out to be very simple. First we rewrite equation (C.2). We introduce the eigenvalues $\lambda_{k}^{+}$and $\lambda_{k}^{-}$

$$
\lambda_{k}^{+}=\left\{\begin{array}{lll}
\lambda_{k} & \text { if } & \lambda_{k}>0 \\
0 & \text { if } & \lambda_{k} \leq 0
\end{array}\right.
$$

and

$$
\lambda_{k}^{-}=\left\{\begin{array}{lll}
0 & \text { if } & \lambda_{k}>0 \\
\lambda_{k} & \text { if } & \lambda_{k} \leq 0
\end{array}\right.
$$

together with the diagonal matrices $\Lambda^{+}=\operatorname{diag}\left\{\lambda_{k}^{+}\right\}, \Lambda^{-}=\operatorname{diag}\left\{\lambda_{k}^{-}\right\}$, which give $|\Lambda|=\Lambda^{+}-\Lambda^{-}$ and $\Lambda=\Lambda^{+}+\Lambda^{-}$. In relation to these diagonal matrices we define $A^{+}=P \Lambda^{+} P^{-1}$ and $A^{-}=$ $P \Lambda^{-} P^{-1}$, which yields $|A|=P|\Lambda| P^{-1}$. Through the construction of the Osher flux from its scalar Engquist and Osher equivalent [16], the Osher flux can be written as

$$
\begin{aligned}
\underline{F}_{(\mathrm{O})}\left(\underline{q}^{\mathrm{L}}, \underline{q}^{\mathrm{R}}\right) & =\underline{f}\left(\underline{q}^{\mathrm{L}}\right)+\int_{\underline{q}^{\mathrm{L}}}^{\underline{q}^{\mathrm{R}}} A^{-} d \underline{q} \\
& =\underline{f}\left(\underline{q}^{\mathrm{R}}\right)-\int_{\underline{q}^{\mathrm{L}}}^{\underline{q}^{\mathrm{R}}} A^{+} d \underline{q} .
\end{aligned}
$$

These representations reveal the upwind character of the Osher flux. More precisely, expression (C.4) states that the flux $\underline{f}\left(\underline{q}^{\mathrm{L}}\right)$, corrected with the characteristic information moving in from 
the right side of the boundary, approximates the flux at this boundary. Note that this characteristic information corresponds with the matrix $A^{-}$. Conversely, the flux $f\left(q^{\mathrm{R}}\right)$ corrected with the characteristic information moving in from the left side of the boundary, also approximates the flux at this boundary. In that case the characteristic information corresponds with the matrix $A^{+}$. Henceforth, we will work with representation (C.5) instead of (C.2), which amounts to evaluation of the following integral along each subcurve $\Gamma_{k}$,

$$
\int_{\Gamma_{k}} A^{+} d \underline{q}
$$

Let us simplify the integral (C.6) by using the parameterization of subcurve $\Gamma_{k}$. This yields

$$
\int_{\Gamma_{k}} A^{+} d \underline{q}=\int_{0}^{s_{k}} P \Lambda^{+} P^{-1} \underline{r}_{k} d s=\int_{0}^{s_{k}} \lambda_{k}^{+} \underline{r}_{k} d s .
$$

Through this formulation we can show that calculation of the Osher flux requires no more than a few flux evaluations. However, we first need to know on which parts of the subcurves $\Gamma_{k}$ the corresponding eigenvalues $\lambda_{k}$ are positive.

For that purpose, we make some assumptions about the eigenvalues $\lambda_{k}$. These assumptions are valid for most physical systems of equations. They also hold for our system of shallow water equations, as we will prove in subsection C.1. The eigenvalue $\lambda_{k}$ is supposed to be either linearly degenerate, which means that along subcurve $\Gamma_{k}$

$$
\frac{d}{d s} \lambda_{k}=\nabla \lambda_{k} \cdot \underline{r}_{k} \equiv 0,
$$

or genuinely non-linear, which means that along subcurve $\Gamma_{k}$

$$
\frac{d}{d s} \lambda_{k}=\nabla \lambda_{k} \cdot \underline{r}_{k} \neq 0
$$

The first case indicates that the eigenvalue $\lambda_{k}$ is constant on $\Gamma_{k}$, i.e., $\lambda_{k}^{+}$is either zero or $\lambda_{k}$ on $\Gamma_{k}$. In the second case the eigenvalue is strictly monotone on $\Gamma_{k}$, which indicates that $\lambda_{k}$ changes sign at most once on $\Gamma_{k}$. We will call the point, $\underline{q}_{s}^{k}$, where this possible change occurs a sonic point. Note that under our assumptions, $\lambda_{k}$ is positive on at most one part of the subcurve $\Gamma_{k}$. When $\underline{q}_{b+}^{k}$ and $\underline{q}_{e+}^{k}$ denote the begin and end points of this part, we find in terms of our Osher path that these points are either $\underline{q}^{\mathrm{L}}, \underline{q}^{\mathrm{R}}, \underline{q}^{1 / 3}, \underline{q}^{2 / 3}$, or $\underline{q}_{\mathrm{s}}^{k}$.

Sofar we have not defined the exact values of the intersection states, $\underline{q}^{1 / 3}, q^{2 / 3}$, and possible $\underline{q}_{\mathrm{s}}^{k}$. We will now attend to this topic. Therefore, we need the concept of Riemann invariants. For each $k$, these invariants $\psi_{\nu}^{k}, \nu \neq k$, are defined as the two independent solutions of the equation

$$
\left(\frac{\partial \psi}{\partial q_{1}}, \frac{\partial \psi}{\partial q_{2}}, \frac{\partial \psi}{\partial q_{3}}\right) \cdot \underline{r}_{k}=0
$$

This definition implies that the invariants $\psi_{\nu}^{k}$ are constant on $\Gamma_{k}$. It is this property that provides us, along our Osher path, with just enough equations to determine the 6 unknown state variables of $\underline{q}^{1 / 3}$ and $\underline{q}^{2 / 3}$. On subcurve $\Gamma_{1}$ we have

$$
\begin{aligned}
& \psi_{2}^{1}\left(\underline{q}^{1 / 3}\right)=\psi_{2}^{1}\left(\underline{q}^{\mathrm{L}}\right), \\
& \psi_{3}^{1}\left(\underline{q}^{1 / 3}\right)=\psi_{3}^{1}\left(\underline{q}^{\mathrm{L}}\right),
\end{aligned}
$$

on subcurve $\Gamma_{2}$,

$$
\begin{aligned}
& \psi_{1}^{2}\left(\underline{q}^{1 / 3}\right)=\psi_{1}^{2}\left(\underline{q}^{2 / 3}\right), \\
& \psi_{3}^{2}\left(\underline{q}^{1 / 3}\right)=\psi_{3}^{2}\left(\underline{q}^{2 / 3}\right),
\end{aligned}
$$


and on subcurve $\Gamma_{3}$,

$$
\begin{aligned}
& \psi_{1}^{3}\left(\underline{q}^{2 / 3}\right)=\psi_{1}^{3}\left(\underline{q}^{\mathrm{R}}\right), \\
& \psi_{2}^{3}\left(\underline{q}^{2 / 3}\right)=\psi_{2}^{3}\left(\underline{q}^{\mathrm{R}}\right) .
\end{aligned}
$$

When a sonic point occurs, we also need the aid of the Riemann invariants. Assume that the eigenvalue $\lambda_{k}$ is genuinely non-linear and remember that $\underline{q}_{\mathrm{b}}^{k}$ and $\underline{q}_{\mathrm{e}}^{k}$ denote respectively the begin and end point of subcurve $\Gamma_{k}$. Note that along our Osher path, the begin and end points of each subcurve $\Gamma_{k}$ are known. Further assume that for subcurve $\Gamma_{k}$ the following inequality holds

$$
\lambda_{k}\left(\underline{q}_{\mathrm{b}}^{k}\right) \cdot \lambda_{k}\left(\underline{q}_{\mathrm{e}}^{k}\right) \leq 0 .
$$

In other words, on $\Gamma_{k}$, a sonic point $\underline{q}_{\mathrm{s}}^{k}$ will be found. To determine the state variable $\underline{q}_{\mathrm{s}}^{k}$, we need at least three equations. The first two equations are provided by the Riemann invariants. We have

$$
\psi_{\nu}^{k}\left(\underline{q}_{\mathrm{b}}\right)=\psi_{\nu}^{k}\left(\underline{q}_{\mathrm{s}}^{k}\right)
$$

where $\nu \neq k$. The third equation follows through the definition of a sonic point,

$$
\lambda_{k}\left(\underline{q}_{\mathrm{s}}^{k}\right)=0 .
$$

Now, we have enough information to calculate integral (C.6) for each subcurve $\Gamma_{k}$. When our system fulfills the conditions on the eigenvalues $\lambda_{k}$, the begin and end state of the part of each subcurve $\Gamma_{k}$ along which the corresponding eigenvalue $\lambda_{k}$ remains positive, i.e., $\underline{q}_{\mathrm{b}+}^{k}\left(s_{\mathrm{b}+}^{k}\right)$ and $\underline{q}_{\mathrm{e}+}^{k}\left(s_{\mathrm{e}+}^{k}\right)$, are known. In that case, the evaluation of the integral (C.6) reduces to at most two flux evaluations pro subcurve, i.e.

$$
\left.\int_{\Gamma_{k}} A^{+} d \underline{q}=\int_{0}^{s_{k}} \lambda_{k}^{+} \underline{r}_{k} d s=\int_{s_{\mathrm{b}+}^{k}}^{s_{\mathrm{e}+}^{k}} \lambda_{k} \underline{r}_{k} d s=\int_{s_{\mathrm{b}+}^{k}}^{s_{\mathrm{e}+}^{k}} A \underline{r}_{k} d s=\int_{\underline{q}_{\mathrm{b}+}^{k}}^{\underline{q}_{\mathrm{e}+}^{k}} \frac{d \underline{f}}{d \underline{q}} d \underline{q}=\underline{f}\left(\underline{q}_{\mathrm{e}+}^{k}\right)-\underline{f}_{\underline{q}_{\mathrm{b}+}^{k}}^{k}\right) .
$$

\section{C.1 The Osher flux for the Shallow Water Equations.}

We have described the construction of the Osher flux and its P-variant Osher path for a general hyperbolic system of equations in one dimension. We now move on to a more precise elaboration, concerning the system of 2D Shallow Water Equations. Our interest will be directed to its spherical formulation. However, we note that, though with different variables, the construction of the Osher flux in case of the stereographic formulation runs along the same lines.

It suffices to approximate the flux $\underline{f}$ on a boundary in the (local) longitudinal direction, i.e.

$$
\underline{f}(\underline{q})=\left(\begin{array}{c}
q_{2} \\
q_{2}^{2} / q_{1}+\frac{1}{2} g q_{1}^{2} \\
\left(q_{2} q_{3}\right) / q_{1}
\end{array}\right)=\left(\begin{array}{c}
H u \\
H u^{2}+\frac{1}{2} g H^{2} \\
H u v
\end{array}\right),
$$

where $\underline{q}=(H, H u, H v)$ denotes the state variable. We apply the Osher flux (C.5) in combination with its P-variant Osher path, see Figure 15. Following the foregoing, we thus use the steps described below for the construction of the Osher flux,

1. Check whether or not the system of equations is hyperbolic. If so, determine the Riemann invariants and construct the P-variant Osher path, i.e., find $q^{1 / 3}$ and $q^{2 / 3}$.

2. Check whether or not the eigenvalues are linearly degenerate or genuinely non-linear. If so, relate these properties to their corresponding subcurves on the Osher path.

3. Check whether or not a sonic point is located on the subcurves corresponding to the genuinely non-linear eigenvalues. If so, calculate the corresponding states.

4. Determine along which parts of the subcurves the corresponding eigenvalues remain positive.

5. The Osher flux can then be found by combining equation (C.5), the P-variant Osher path and the parts found in step 4. 
Step 1 The Jacobian matrix $A$ of the flux $\underline{f}$ with respect to $\underline{q}=(H, H u, H v)$ reads

$$
A=\frac{d \underline{f}}{d \underline{q}}=\left(\begin{array}{ccc}
0 & 1 & 0 \\
\frac{-q_{2}^{2}}{q_{1}^{2}}+g q_{1} & \frac{2 q_{2}}{q_{1}} & 0 \\
\frac{-q_{2} q_{3}}{q_{1}^{2}} & \frac{q_{3}}{q_{1}} & \frac{q_{2}}{q_{1}}
\end{array}\right)=\left(\begin{array}{ccc}
0 & 1 & 0 \\
-u^{2}+g H & 2 u & 0 \\
-u v & v & u
\end{array}\right) .
$$

Its eigenvalues are given by

$$
\begin{aligned}
& \lambda_{1}=u-\sqrt{g H}, \\
& \lambda_{2}=u, \\
& \lambda_{3}=u+\sqrt{g H},
\end{aligned}
$$

with corresponding eigenvectors,

$$
\begin{aligned}
& \underline{r}_{1}=(1, u-\sqrt{g H}, v)^{T}, \\
& \underline{r}_{2}=(0,0,1)^{T}, \\
& \underline{r}_{3}=(1, u+\sqrt{g H}, v)^{T},
\end{aligned}
$$

establishing that our system of equations is hyperbolic. Note that the eigenvalues are numbered in increasing order.

The Riemann invariants follow after solving equation (C.9) for each subcurve $\Gamma_{k}$,

$$
\begin{array}{ll}
\psi_{2}^{1}=v, & \psi_{3}^{1}=u+2 \sqrt{g H}, \\
\psi_{1}^{2}=H, & \psi_{3}^{2}=H u, \\
\psi_{1}^{3}=v, & \psi_{2}^{3}=u-2 \sqrt{g H} .
\end{array}
$$

The P-variant Osher path is then illustrated in Figure 16. The eigenvalues indicate the propagation speeds along the corresponding characteristic directions, i.e. along the corresponding eigenvectors $\underline{r}_{k} \cdot \underline{q}^{1 / 3}$ and $\underline{q}^{2 / 3}$ result after solving system (C.10)-(C.12)

$$
\underline{q}^{\frac{1}{3}}=\left(\begin{array}{c}
H_{\frac{1}{3}} \\
H_{\frac{1}{3}} u_{\frac{1}{3}} \\
H_{\frac{1}{3}} v_{\mathrm{L}}
\end{array}\right), \quad \underline{q}^{\frac{2}{3}}=\left(\begin{array}{c}
H_{\frac{1}{3}} \\
H_{\frac{1}{3}} u_{\frac{1}{3}} \\
H_{\frac{1}{3}} v_{\mathrm{R}}
\end{array}\right)
$$

where

$$
\begin{aligned}
& H_{\frac{1}{3}}=\frac{1}{16} \frac{1}{g}\left(\left(u_{\mathrm{L}}-u_{\mathrm{R}}\right)+2\left(\sqrt{g H_{\mathrm{L}}}+\sqrt{g H_{\mathrm{R}}}\right)\right)^{2}, \\
& u_{\frac{1}{3}}=\frac{1}{2}\left(u_{\mathrm{L}}+u_{\mathrm{R}}\right)+\sqrt{g H_{\mathrm{L}}}-\sqrt{g H_{\mathrm{R}}} .
\end{aligned}
$$

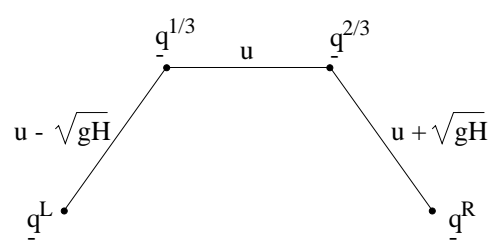

Figure 16: A schematic representation of the P-variant Osher path $\Gamma$ in case of the SWEs. 
Step 2 Elaborating the expressions (C.7) and (C.8), we find that the eigenvalue $\lambda_{2}$ is linearly degenerate and the eigenvalues $\lambda_{1}$ and $\lambda_{3}$ are genuinely non-linear. We have

$$
\nabla_{\underline{q}} \lambda_{2} \cdot \underline{r}_{2}=-\frac{U}{H} \cdot 0+\frac{1}{H} \cdot 0=0, \quad \forall \underline{q} \in S,
$$

where $S$ defines the state space $S=\left\{\underline{q}: q_{1} \in \mathbb{R}^{+}, q_{2} \in \mathbb{R}, q_{3} \in \mathbb{R}\right\}$ and

$$
\begin{aligned}
& \nabla_{\underline{q}} \lambda_{1} \cdot \underline{r}_{1}=\left(-\frac{U}{H}-\frac{1}{2} \sqrt{\frac{g}{H}}\right) \cdot 1+\frac{1}{H} \cdot(u-\sqrt{g H})=-\frac{3}{2} \sqrt{\frac{g}{H}} \neq 0, \quad \forall \underline{q} \in S, \\
& \nabla_{\underline{q}} \lambda_{3} \cdot \underline{r}_{3}=\left(-\frac{U}{H}+\frac{1}{2} \sqrt{\frac{g}{H}}\right) \cdot 1+\frac{1}{H} \cdot(u+\sqrt{g H})=\frac{3}{2} \sqrt{\frac{g}{H}} \neq 0, \quad \forall \underline{q} \in S .
\end{aligned}
$$

Step 3 A sonic point can occur on subcurve $\Gamma_{1}$ or on subcurve $\Gamma_{3}$. When a sonic point is located on subcurve $\Gamma_{1}$, or in other words, when the inequality $\lambda_{1}\left(\underline{q}^{\mathrm{L}}\right) \cdot \lambda_{1}\left(\underline{q}^{1 / 3}\right) \leq 0$ holds, the sonic point is given as

$$
\underline{q}_{\mathrm{s}}^{1}=\left(\begin{array}{c}
H_{\mathrm{s}} \\
H_{\mathrm{s}} \sqrt{g H_{\mathrm{s}}} \\
H_{\mathrm{s}} v_{\mathrm{L}}
\end{array}\right),
$$

where

$$
H_{\mathrm{s}}=\frac{1}{9 g}\left(u_{\mathrm{L}}+2 \sqrt{g H_{\mathrm{L}}}\right)^{2} .
$$

When a sonic point is located on subcurve $\Gamma_{3}$, i.e., when the inequality $\lambda_{3}\left(\underline{q}^{2 / 3}\right) \cdot \lambda_{3}\left(\underline{q}^{\mathrm{R}}\right) \leq 0$ holds, the sonic point reads

$$
\underline{q}_{\mathrm{s}}^{3}=\left(\begin{array}{c}
H_{\mathrm{s}} \\
-H_{\mathrm{s}} \sqrt{g H_{\mathrm{s}}} \\
H_{\mathrm{s}} v_{\mathrm{R}}
\end{array}\right),
$$

where

$$
H_{\mathrm{s}}=\frac{1}{9 g}\left(u_{\mathrm{R}}-2 \sqrt{g H_{\mathrm{R}}}\right)^{2} .
$$

Step 4 The parts on the subcurves $\Gamma_{k}$ along which the corresponding eigenvalues are positive can be found through the signs of the eigenvalues

$$
\begin{aligned}
& \lambda_{\mathrm{L}}=u_{\mathrm{L}}-\sqrt{g H_{\mathrm{L}}}, \\
& \lambda_{\frac{1}{3}}=u_{\frac{1}{3}}-\sqrt{g H_{\frac{1}{3}}}, \\
& \lambda_{\frac{1}{2}}=u_{\frac{1}{2}}=u_{\frac{1}{3}}, \\
& \lambda_{\frac{2}{3}}=u_{\frac{2}{3}}+\sqrt{g H_{\frac{2}{3}}}=u_{\frac{1}{3}}+\sqrt{g H_{\frac{1}{3}}}, \\
& \lambda_{\mathrm{R}}=u_{\mathrm{R}}+\sqrt{g H_{\mathrm{R}}} .
\end{aligned}
$$

Realizing that we always have $\lambda_{1 / 3} \leq \lambda_{1 / 2} \leq \lambda_{2 / 3}$, we can write down all 16 possible sign combinations of the eigenvalues (C.13) along the Osher path, see Figure 17. The plus and minus signs along the Osher path in clockwise direction indicate the signs of respectively the eigenvalues $\lambda_{\mathrm{L}}$, $\lambda_{1 / 3}, \lambda_{1 / 2}, \lambda_{2 / 3}$, and $\lambda_{\mathrm{R}}$. A crossbar on $\Gamma_{1}$ or $\Gamma_{3}$ indicates the existence of a sonic point. Note that these points are also related to the sign of the eigenvalues (C.13). Respecting the properties of the eigenvalues $\lambda_{1}, \lambda_{2}$, and $\lambda_{3}$, the needed parts along the Osher path are known in case of each different sign combination. 


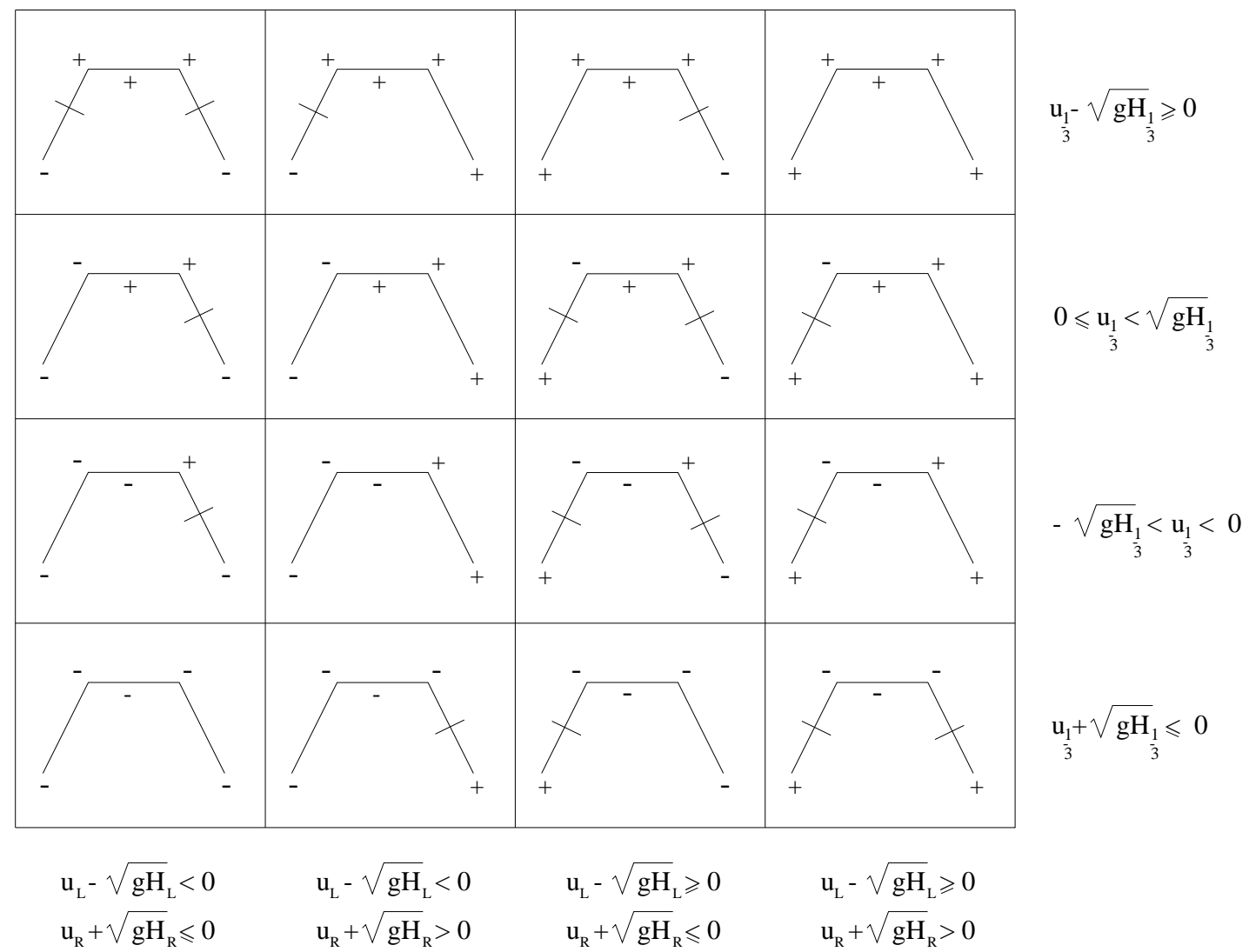

Figure 17: The different sign combinations of the eigenvalues along the Osher path $\Gamma$.

Step 5 We demonstrate the evaluation of the Osher flux for sign combination $(2,1)$, i.e., $\lambda_{\mathrm{L}}<0$, $\lambda_{1 / 3}<0, \lambda_{1 / 2}>0, \lambda_{2 / 3}>0, \lambda_{\mathrm{R}} \leq 0$. The eigenvalues $\lambda_{\mathrm{L}}<0$ and $\lambda_{1 / 3}<0$ indicate that $\lambda_{1}(q)<0$ along subcurve $\Gamma_{1}$. On $\Gamma_{2}$ we have $\lambda_{2}(q)>0$, because $\lambda_{1 / 2}=u_{1 / 2}>0$ and $\lambda_{2}$ is a linearly degenerate eigenvalue, thus constant along $\Gamma_{2}$. On $\Gamma_{3}$ a sonic point occurs. In combination with the fact that $\lambda_{3}$ is linearly degenerate and $\lambda_{2 / 3}>0$, this indicates that along $\Gamma_{3}$, between the states $\underline{q}^{2 / 3}$ and $\underline{q}_{\mathrm{s}}^{3}, \lambda_{3}(\underline{q})>0$. Consequently, the Osher flux reads

$$
\begin{aligned}
\underline{F}\left(\underline{q}^{\mathrm{L}}, \underline{q}^{\mathrm{R}}\right) & =\underline{f}\left(\underline{q}^{\mathrm{R}}\right)-\left(\int_{\Gamma_{1}} A^{+} d \underline{q}+\int_{\Gamma_{2}} A^{+} d \underline{q}+\int_{\Gamma_{3}} A^{+} d \underline{q}\right) \\
& =\underline{f}\left(\underline{q}^{\mathrm{R}}\right)-\left[0+\left(\underline{f}\left(\underline{q}^{\frac{2}{3}}\right)-\underline{f}\left(\underline{q}^{\frac{1}{3}}\right)\right)+\left(\underline{f}\left(\underline{q}_{\mathrm{s}}^{3}\right)-\underline{f}\left(\underline{q}^{\frac{2}{3}}\right)\right)\right] \\
& =\underline{f}\left(\underline{q}^{\mathrm{R}}\right)-\underline{f}\left(\underline{q}_{\mathrm{s}}^{3}\right)+\underline{f}\left(\underline{q}^{\frac{1}{3}}\right) .
\end{aligned}
$$

Elaboration of the Osher flux for the remaining sign combinations yields Table 7 .

In relation to Table 7, we remark the following. Implementation of the Osher flux leads to a succession of different conditional statement evaluations, which is not very efficient. In practice though, we can discard most of the possible sign combinations. We mostly encounter flows, where we have $|\underline{u}|<\sqrt{g H}$, since $H$ represents the depth of the atmosphere, which is always near $10^{4} \mathrm{~m}$ approximately. In that case, Table 7 reduces to Table 8 ,

D. General Formulation OF THE MODIFIED $\kappa$-SCHEME FOR NON-UNIFORM GRIDS In this appendix we give the general formulation of the non-uniform $\kappa$-scheme for different values of $\kappa$. It concerns the non-uniform equivalents of the 3-point $(\kappa=1 / 3)$-scheme, the 2 -point central $(\kappa=1)$-scheme, the 2-point upwind $(\kappa=-1)$-scheme, and the 3 -point upwind $(\kappa=1 / 2)$-scheme. 


\begin{tabular}{|c|c|c|c|c|}
\hline $\begin{array}{l}\underline{f}\left(\underline{q}^{\mathrm{R}}\right)-\underline{f}\left(\underline{q}_{\mathrm{s}}^{3}\right)+ \\
+\underline{f}\left(\underline{q}_{\mathrm{s}}^{1}\right)\end{array}$ & $\underline{f}\left(\underline{q}_{\mathrm{s}}^{1}\right)$ & $\begin{array}{l}\underline{f}\left(\underline{q}^{\mathrm{L}}\right)+ \\
+\underline{f}\left(\underline{q}^{\mathrm{R}}\right)-\underline{f}\left(\underline{q}_{\mathrm{s}}^{3}\right)\end{array}$ & $\underline{f}\left(\underline{q}^{\mathrm{L}}\right)$ & $u_{\frac{1}{3}}-\sqrt{g H_{\frac{1}{3}}} \geq 0$ \\
\hline $\begin{array}{l}\underline{f}\left(\underline{q}^{\mathrm{R}}\right)-\underline{f}\left(\underline{q}_{\mathrm{s}}^{3}\right)+ \\
+\underline{f}\left(\underline{q}^{\frac{1}{3}}\right)\end{array}$ & $\underline{f}\left(\underline{q}^{\frac{1}{3}}\right)$ & $\begin{array}{l}\underline{f}\left(\underline{q}^{\mathrm{L}}\right)+ \\
+\underline{f}\left(\underline{q}^{\mathrm{R}}\right)-\underline{f}\left(\underline{q}_{\mathrm{s}}^{3}\right)+ \\
+\underline{f}\left(\underline{q}^{\frac{1}{3}}\right)-\underline{f}\left(\underline{q}_{\mathrm{s}}^{1}\right)\end{array}$ & $\begin{array}{l}\underline{f}\left(\underline{q}^{\mathrm{L}}\right)+ \\
+\underline{f}\left(\underline{q}^{\frac{1}{3}}\right)-\underline{f}\left(\underline{q}_{\mathrm{s}}^{1}\right)\end{array}$ & $0<u_{\frac{1}{3}}<\sqrt{g H_{\frac{1}{3}}}$ \\
\hline $\begin{array}{l}\underline{f}\left(\underline{q}^{\mathrm{R}}\right)-\underline{f}\left(\underline{q}_{\mathrm{s}}^{3}\right)+ \\
+\underline{f}\left(\underline{q}^{\frac{2}{3}}\right)\end{array}$ & $\underline{f}\left(\underline{q}^{\frac{2}{3}}\right)$ & $\begin{array}{l}\underline{f}\left(\underline{q}^{\mathrm{L}}\right)+ \\
+\underline{f}\left(\underline{q}^{\mathrm{R}}\right)-\underline{f}\left(\underline{q}_{\mathrm{s}}^{3}\right)+ \\
=\underline{f}\left(\underline{q}^{\frac{2}{3}}\right)-\underline{f}\left(\underline{q}_{\mathrm{s}}^{1}\right)\end{array}$ & $\begin{array}{l}\underline{f}\left(\underline{q}^{\mathrm{L}}\right)+ \\
+\underline{f}\left(\underline{q}^{\frac{2}{3}}\right)-\underline{f}\left(\underline{q}_{\mathrm{s}}^{1}\right)\end{array}$ & $-\sqrt{g H_{\frac{1}{3}}}<u_{\frac{1}{3}}<0$ \\
\hline$\underline{f}\left(\underline{q}^{\mathrm{R}}\right)$ & $\underline{f}\left(\underline{q}_{\mathrm{s}}^{3}\right)$ & $\begin{array}{l}\underline{f}\left(\underline{q}^{\mathrm{L}}\right)+ \\
+\underline{f}\left(\underline{q}^{\mathrm{R}}\right)-\underline{f}\left(\underline{q}_{\mathrm{s}}^{1}\right) \\
\end{array}$ & $\begin{array}{l}\underline{f}\left(\underline{\mathrm{L}}^{\mathrm{L}}\right)+ \\
+\underline{f}\left(\underline{q}_{\mathrm{s}}^{3}\right)-\underline{f}\left(\underline{q}_{\mathrm{s}}^{1}\right) \\
\end{array}$ & $u_{\frac{1}{3}}+\sqrt{g H_{\frac{1}{3}}} \leq 0$ \\
\hline$u_{L}-\sqrt{g H_{L}}<0$ & $u_{L}-\sqrt{g H_{L}}<0$ & $u_{L}-\sqrt{g H_{L}} \geq 0$ & $u_{L}-\sqrt{g H_{L}} \geq 0$ & \\
\hline$u_{R}+\sqrt{g H_{R}} \leq 0$ & $u_{R}+\sqrt{g H_{R}}>0$ & $u_{R}+\sqrt{g H_{R}} \leq 0$ & $u_{R}+\sqrt{g H_{R}}>0$ & \\
\hline
\end{tabular}

Table 7: The Osher flux depending on the signs of the eigenvalues $\lambda_{L}, \lambda_{\frac{1}{3}}, \lambda_{\frac{1}{2}}, \lambda_{\frac{2}{3}}$ and $\lambda_{R}$.

\begin{tabular}{|c||c|}
\hline$\underline{f}\left(\underline{q}^{\frac{1}{3}}\right)$ & $0<u_{\frac{1}{3}}<\sqrt{g H_{\frac{1}{3}}}$ \\
\hline$\underline{f}\left(\underline{q}^{\frac{2}{3}}\right)$ & $-\sqrt{g H_{\frac{1}{3}}}<u_{\frac{1}{3}}<0$ \\
\hline \hline $\begin{array}{l}u_{L}-\sqrt{g H_{L}}<0 \\
u_{R}+\sqrt{g H_{R}}>0\end{array}$ & \\
\hline
\end{tabular}

Table 8: Reduction of Table 7 under the assumption $|\underline{u}|<\sqrt{g H}$.

Let $\underline{q}_{\text {? }}$ be the unknown state variable to be found by $1 \mathrm{D}$ state interpolation in a certain direction, say $x$. Let $\ell_{i}$ denote the cell width of a cell $i$ in $x$-direction and let $\underline{q}_{i}$ denote the state variable in its cell center, see Figure 18, where we use $\ell_{1}, \ell_{2}$ etc. for convenience of notation. The modified $\kappa$-scheme is now given as a function $I_{\kappa}$ with arguments $\underline{q}_{1}, \ell_{1}, \underline{q}_{2}, \ell_{2}$ etc. based on Figure 18 . The modified $(\kappa=1 / 3)$-scheme then reads

$$
I_{\frac{1}{3}}\left(\underline{q}_{1}, \underline{q}_{2}, \underline{q}_{3}, \ell_{0}, \ell_{1}, \ell_{2}, \ell_{3}, \ell_{4}\right)=\alpha \underline{q}_{1}+\beta \underline{q}_{2}+\gamma \underline{q}_{3}
$$

with

$$
\begin{aligned}
\alpha & =-2 * \frac{\ell_{3}\left(\ell_{2} \ell_{3}+\ell_{2} \ell_{4}+\ell_{3}^{2}+\ell_{3} \ell_{4}\right)}{\left(\ell_{1}^{3}+\ell_{1}^{2}\left(5 \ell_{2}+3 \ell_{3}+\ell_{4}\right)+\ell_{1}\left(8 \ell_{2}^{2}+9 \ell_{2} \ell_{3}+3 \ell_{2} \ell_{4}+2 \ell_{3}^{2}+\ell_{3} \ell_{4}\right)+4 \ell_{2}^{3}+\ell_{2}^{2}\left(6 \ell_{3}+2 \ell_{4}\right)+\ell_{2}\left(2 \ell_{3}^{2}+\ell_{3} \ell_{4}\right)\right)}, \\
\beta & =1-\alpha-\gamma, \\
\gamma & =2 * \frac{\ell_{2}\left(\ell_{0} \ell_{1}+\ell_{0} \ell_{2}+2 \ell_{1}^{2}+3 \ell_{1} \ell_{2}+\ell_{2}^{2}\right)}{\left(\ell_{0}+2 \ell_{1}+2 \ell_{2}+\ell_{3}\right)\left(\ell_{1} \ell_{2}+\ell_{1} \ell_{3}+2 \ell_{2}^{2}+3 \ell_{2} \ell_{3}+\ell_{3}^{2}\right)} .
\end{aligned}
$$




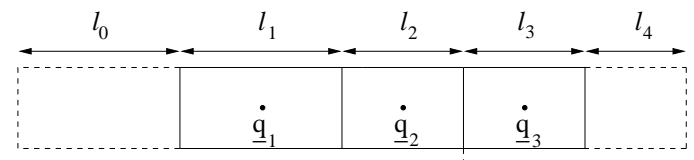

$\underline{\mathrm{q}}_{?}$

Figure 18: General situation around a cell boundary. $\underline{q}_{?}$ is the unknown state variable to be found by interpolation.

The modified 2-point central ( $\kappa=1)$-scheme, the 2-point upwind $(\kappa=-1)$-scheme, and the 3 -point upwind $(\kappa=1 / 2)$-scheme are

$$
\begin{array}{ll}
I_{1}\left(\underline{q}_{2}, \underline{q}_{3}, \ell_{2}, \ell_{3}\right) & =\frac{\ell_{3}}{\ell_{2}+\ell_{3}} \underline{q}_{2}+\frac{\ell_{2}}{\ell_{2}+\ell_{3}} \underline{q}_{3}, \\
I_{-1}\left(\underline{q}_{1}, \underline{q}_{2}, \ell_{1}, \ell_{2}\right) & =\frac{-\ell_{2}}{\ell_{1}+\ell_{2}} \underline{q}_{1}+\frac{\ell_{1}+2 \ell_{2}}{\ell_{1}+\ell_{2}} \underline{q}_{2}, \\
I_{\frac{1}{2}}\left(\underline{q}_{1}, \underline{q}_{2}, \underline{q}_{3}, \ell_{1}, \ell_{2}, \ell_{3}\right) & =-\frac{\ell_{2} \ell_{3}}{\left(\ell_{1}+2 \ell_{2}+\ell_{3}\right)\left(\ell_{1}+\ell_{2}\right)} \underline{q}_{1}+\frac{\left(\ell_{1}+2 \ell_{2}\right) \ell_{3}}{\left(\ell_{2}+\ell_{3}\right)\left(\ell_{1}+\ell_{2}\right)} \underline{q}_{2}+\frac{\ell_{2}\left(\ell_{1}+2 \ell_{2}\right)}{\ell_{1} \ell_{2}+\ell_{1} \ell_{3}+2 \ell_{2}^{2}+3 \ell_{2} \ell_{3}+\ell_{3}^{2}} \underline{q}_{3} .
\end{array}
$$

